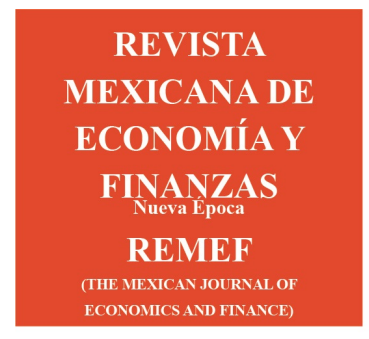
Revista Mexicana de Economía y Finanzas Nueva Época
Volumen 15 Segundo Número Especial Aniversario Agosto $2020 \quad$ pp. 551-576
DOI: https://doi.org/10.21919/remef .v15i0.546
(Recibido: 13/abril/2020, aceptado: 13/agosto/2020)

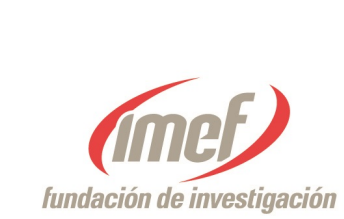

SNEA - Entrepreneurial research in emerging economies: recent advances Editor Invitado: Dr. Samuel Mongrut

\title{
Orientación Emprendedora y Emprendimiento Corporativo: Diferencias y complementariedad en un modelo intención-acción
}

\author{
Raúl Armando Cardona Montoya ${ }^{1}$ \\ Universidad EAFIT, Colombia \\ Izaias Martins ${ }^{2}$ \\ Universidad EAFIT, Colombia \\ Hermilson Velásquez Ceballos ${ }^{3}$ \\ Universidad EAFIT, Colombia
}

\section{Resumen}

El objetivo del estudio es explicar diferencias y complementariedad entre la Orientación emprendedora como intención y el Emprendimiento corporativo, fenómenos que se tratan de forma indiferente por diversos autores. Mediante encuesta y datos de 137 PYMEs manufactureras, con un modelo de análisis multivariante y ecuaciones estructurales, se analiza la relación entre los dos fenómenos, poco contrastada cuantitativamente en estudios previos, mediada por las capacidades de gestión. A diferencia de los trabajos que analizan una sola capacidad como determinante, se analizan cuatro, entre ellas la de financiación, poco explorada y otra que incluso no se encontró en estudios previos, la Capacidad de Evaluar y gestionar proyectos, pero de alto impacto en la renovación empresarial, por lo que representa novedad. Los resultados confirman la existencia la relación positiva entre los dos constructos estudiados e igual de la orientación emprendedora con las capacidades de gestión analizadas. Como limitaciones se incluye la deficiente estructura organizacional de las PYMEs y el dejar por fuera otras capacidades como la cultura organizacional, estilos de liderazgo, innovación organizacional y otras variables, que en interacción pueden dinamizar la innovación y competitividad de las PYMEs.

Clasificación JEL: M100, O30, O32, C13

Palabras claves: Orientación emprendedora, emprendimiento corporativo, $R B V$, capacidades de emprendimiento corporativo, absorción de conocimiento, redes externas de cooperación, evaluación y gestión de proyectos

\section{Entrepreneurial Guidance and Corporate Entrepreneurship: Differences and complementarity in an intent-action model}

\section{Abstract}

The objective of this study is to analyze differences and complementarity between entrepreneurial orientation

\footnotetext{
${ }^{1}$ Carrera 49 No. 7 Sur-50 Medellín, Colombia. rcardona@eafit.edu.co. Teléfono 0543164781568 http://190.216.132.131: $8081 /$ cvlac/visualizador/generarCurriculoCv.do?cod_rh $=0000149985$

${ }^{2}$ Carrera 49 No. 7 Sur-50 Medellín, Colombia. imartins6@eafit.edu.co Teléfono 054 2619500. http://190.216.132.131:8081/ cvlac/visualizador/generarCurriculoCv.do?cod_rh=0000007454

${ }^{3}$ Carrera 49 No. 7 Sur-50 Medellín, Colombia. Teléfono 054 2619500. evelas@eafit.edu.co http://190.216.132.131:8081/ cvlac/visualizador/generarCurriculoCv.do?cod_rh=0000150835

*Fuente de financiamiento: declarado en agradecimientos
} 


\section{Abstract}

as intention and corporate entrepreneurship, phenomena that are treated indifferently by different authors. A survey of 137 manufacturing SMEs was carried out, estimating multivariate model and structural equations, in order to analysis the relationship between the two phenomena, little quantitatively contrasted in previous studies. Management capabilities drive the complementarity between the two constructs. Unlike the works that study a single capacity as a determinant, four are analyzed, among them that of financing, little explored and another that was not even found in previous studies, the ability to evaluate and manage projects, but with high impact on the business renewal, representing novelty. The results confirm the existence of a positive relationship between the two constructs studied and an equal entrepreneurial orientation with the analyzed management capabilities. Limitations include the poor organizational structure of SMEs and leaving out other capabilities such as organizational culture, leadership styles, management innovation and other variables, which in interaction can stimulate innovation and competitiveness of SMEs.

JEL Classification: M100, О30, О32, C13

Keywords: Entrepreneurial orientation, corporate entrepreneurship, corporate entrepreneurships capabilities, absorptive knowledge, external cooperation networks, evaluation and project management

\section{Introducción}

En la revisión de literatura sobre Emprendimiento Corporativo -EC, se observa que el fenómeno ha sido extensamente estudiado por más de cuatro décadas, evolucionado en sus dimensiones, determinantes, y definiciones. Una de las perspectivas de estudio del EC, cada vez creciente, es la actitud emprendedora de las organizaciones, denominada Orientación Emprendedora -OE (Miller, 1983; Covin y Slevin, 1991.

Se ha establecido que las distintas acciones emprendedoras en las que se materializa el EC, son un claro reflejo de la OE de las organizaciones (Stambaugh, Martinez, Lumpkin y Kataria (2017), y en estudios separados de cada uno de los fenómenos se confirma que están positivamente relacionados con el desempeño de la empresa. El estudio separado, obedece a que tienen algunos determinantes comunes, y diversos autores los tratan como sinónimos o los usan de manera indistinta (Covin y Slevin, 1991; Lumpkin y Dess, 1996; Covin y Wales 2019). Tácitamente, así lo señalan Urbano, Toledano y Soriano (2010, p.118): "La orientación emprendedora que adoptan algunas organizaciones constituye lo que se identifica en la literatura internacional como corporate entrepreneurship, intrapreneurship o corporate venturing". De forma crítica, Covin y Lumpkin (2011) preguntan si la EO significa disposición o comportamiento (acciones), reflexión que abrió posibilidades de estudiarla por separado del EC.

Dada la complejidad de los dos fenómenos y los factores que les son comunes, este trabajo apunta a contribuir en la aclaración de diferencias, así como de la complementariedad existente, interés de investigación en los últimos años para cerrar las brechas que existen entre la intención de emprender y acciones concretas en que se manifiesta esta actitud (Hasan, Syyedhamzeh y Ali, 2013; Schlaegel y Koenig, 2014; Gelderen, Kautonen y Fink, 2015), y analizada más en la creación de empresas. Se analiza el significado de OE y

EC, haciendo referencia al primero de ellos como la actitud emprendedora, voluntad e intención firme de buscar nuevas ideas de negocio o acciones emprendedoras, que para lograrse requieren condiciones y recursos específicos.

La actitud emprendedora tiene sus raíces teóricas en los trabajos de Mintzberg (1994), Miller (1983), posteriormente denominada postura emprendedora por Covin y Slevin (1991) y orientación emprendedora por Lumpkin y Dess (1996); estos últimos autores entienden el fenómeno como la intención previamente existente para que el EC se desarrolle al interior de las organizaciones, por lo que puede entenderse la importancia de su complementariedad.

Bohr (como se citó en Blanco y Pirela, 2015), explica el concepto de complementariedad como principio 
que involucra distintos fenómenos, con ciertas características o propiedades que no se observan en ambos de manera simultánea, posibilitando visiones complementarias e importantes para intentar dar una explicación completa de una misma realidad.

Strathern (como se citó en Blanco y Pirela, 2015), señala que las evidencias que se obtienen bajo diferentes condiciones, deben considerarse complementarias, ya que sólo la totalidad de los fenómenos agota la información posible del objeto de estudio. Desde una perspectiva operativa, la complementariedad se usa en este trabajo como estrategia de integración del emprendimiento con los dos enfoques o constructos requeridos, esperándose, como se plantea en las hipótesis, que el desarrollo de la OE puede llevar a un mejor desempeño del EC, en especial cuando se estructuran procesos y capacidades que interactúan e impulsan esta relación.

Bouchard y Basso (2011) plantean que la relación OE y EC no se ha explorado suficientemente, que ambos enfoques conceptuales se han desarrollado en paralelo, con poca o ninguna conexión; presentando una proposición teórica entre EO; sus proposiciones teóricas planteadas sobre la su relación directa y positiva, dieron posibilidades de estudios en el mismo sentido, para profundizar en su diferenciación y complementariedad (Goodale, Kuratko, Hornsby y Covin, 2011; Hasan, Syyedhamzeh y Ali, 2013). Igual Todorovic, Todorovic y Ma (2015), afirman que se ha avanzado en la definición y dimensiones que componen la OE como campo de desarrollo del EC, pero que existe poca discusión que incluya ambos constructos. Titus Jr, Parker y Covin (2019) afirman que mientras que la literatura de OE ha avanzado en desarrollar diferentes conceptualizaciones, aún hay muchas discrepancias entre la postura emprendedora y los resultados en el emprendimiento y el desempeño empresarial.

Para Wales (2016), el concepto de EO ha motivado una gran cantidad de estudios. Existen diversas definiciones de OE, una de las definiciones de mayor aceptación (Lumpkin y Dess, 1996, p.137): "Procesos, prácticas y toma de decisiones sobre actividades dirigidas a nuevos negocios, caracterizadas por una o más de las siguientes cinco dimensiones: la propensión a actuar autónomamente, la voluntad de innovar y tomar riesgos, la tendencia a la agresividad hacia los competidores y la proactividad relativa a las oportunidades del mercado".

De otro lado, sobre el EC, Sharma y Chrisman (1999, p.13) lo definen así: "el proceso en el que un individuo o grupo de individuos en asociación, crean una nueva organización, la renuevan o innovan". Para explicar la forma en que se materializa el EC, se han usado distintos términos: Intraemprendimiento, desarrollado y popularizado por Pinchot (como se citó en Antoncic y Hisrich, 2001; Bouchard y Basso, 2011); proyectos innovadores, (Urbano, Toledano y Soriano (2010); actividades de innovación (Mcfadzean, O'Loughlin y Shaw, 2005; Hasan, Syyedhamzeh y Ali, 2013). Los términos se derivan de la teoría económica de la destrucción creativa (Schumpeter, 1942) y de la definición de innovación de la OCDE en el manual de OSLO (2005).

Corbett, Covin, O'Connor y Tucci (2013) afirman que en los últimos años, el estudio del EC han apuntan a tratar la EO y el EC como fenómenos de desarrollo teórico paralelo. Todorovic, Todorovic y Ma (2015) señalan que hay espacio para que nuevas investigaciones analicen los dos constructos en interdependencia y Covin y Wales (2019) señalan que aunque ambos fenómenos tienen dimensiones comunes con las cuales se les ha tratado de explicar, debe avanzar el debate sobre sus diferencias y complementos.

Estos retos planteados por los anteriores autores, generan espacios de investigación, explorados conjuntamente en este trabajo, más si se tiene en cuenta que son escasos los trabajos han contrastado cuantitativamente esta relación. Hasan, Syyedhamzeh y Ali (2013) validan estadísticamente la relación EO y los resultados del EC, enfocados como acciones innovadoras; Gelderen, Kautonen y Fink (2015) analizan la brecha y relación entre EO como intención y acciones, en relación a la creación de empresas, como otra perspectiva del emprendimiento de amplio interés. Titus Jr, Parker y Covin (2019) analizan las discrepancias de la postura estratégica de una empresa y en los logros en acciones enfocadas a la creación de nuevas unidades de negocios o negocios diversos, fusiones y adquisiciones, $\mathrm{I}+\mathrm{D}$, entre otros. 
REMEF (The Mexican Journal of Economics and Finance)

Covin y Wales (2019), continúan la discusión y debate sobre este asunto central: ¿Cómo se debe conceptualizar la OE?, ¿Cómo se relaciona con otros constructos similares que colectivamente comprenden el dominio del EC? Los autores advierten que se mantiene el desafío conceptual central responder a estas preguntas, sobre lo que significa para una empresa ser ${ }^{\mathrm{em}}$ prendedora", porque puede implicar muchas cosas diferentes, siendo este el reto de este trabajo.

El interés en el EC, corresponde a los impactos en el crecimiento de las organizaciones, su renovación, innovación, internacionalización, mejoras en desempeño y logro de ventajas competitivas sostenibles (Covin y Slevin, 1991; Zahra, 1993; Ireland, Kuratko y Morris, 2006; Kuratko y Audretsch, 2009), registrándose un alto número de los estudios que han analizado sus antecedentes y consecuencias. Sobre las primeras, se encuentran en primer lugar, los factores externos, como el dinamismo del entorno y rivalidad competitiva (Covin y Slevin, 1991; Zahra, 1993); de otro lado, están los determinantes internos de la organización, fundamentados por la Teoría de Recursos y Capacidades -RBV (Wernerfelt, 1984; Barney, 1991; Teece, Pisano y Schuen, 1997; Baker, Grinstein y Perin, 2019; Taheri, Bititci, Gannon y Cordina, 2019).

Es en esta perspectiva y en la Teoría RBV se fundamenta este trabajo, que tiene como objetivo explicar diferencias y complementariedad entre OE y EC y contrastarlas además en su relación de complementariedad, mediada por distintas capacidades de gestión, algunas usadas para analizar en separado cada fenómeno.

Para Zahra (1993), el emprendimiento innovador se materializa en acciones que pueden considerarse como innovadoras, que mejorar la posición competitiva de una empresa y el desempeño financiero. Innovación se estableció por Schumpeter (1942), y en este sentido amplio se fundamentó la definición que ofrece el Manual de Oslo (OCDE, 2005, p.56):

"Una innovación es la introducción de un nuevo, o significativamente mejorado, producto (bien o servicio), de un proceso, de un nuevo método de comercialización o de un nuevo método organizativo, en las prácticas internas de la empresa, la organización del lugar de trabajo o las relaciones exteriores".

Esta amplia definición integra un conjunto amplio de acciones emprendedoras (innovaciones, de intraemprendimiento, proyectos innovadores, necesarias para que las empresas se transformen permanentemente y enfrenten los retos de la globalización y la Cuarta Revolución Industrial, en materia de innovación y competitividad (Banco Mundial, Doing Business, 2019; GEM, 2018). Diversos organismos multilaterales, afirman que las naciones alrededor del mundo enfrentan retos de diseño de políticas públicas, orientadas a dinamizar el emprendimiento, la innovación, generar empleo, mejorar la competitividad, impulsar el desarrollo económico y social. Múltiples regulaciones apuntan a impulsar la creación de empresas, el emprendimiento en las organizaciones establecidas y la innovación.

El Reporte Global de innovación "Energizando al mundo con innovación" (FEM - Índice Global de Innovación, 2018) y (Banco Mundial- Doing Business, 2019, afirman que las regulaciones empresariales son muy diversas entre países, y en algunos casos pueden ser excesivas y desincentivan el espíritu empresarial, el crecimiento del sector privado, causan pobreza y obstaculizan la prosperidad compartida, A su vez, el exceso de regulación puede dar lugar a corrupción e injerencia excesiva del Estado, que debe complementar (no sustituir) al mercado.

Metodológicamente se utiliza un enfoque cuantitativo fundamentado en técnicas de análisis multivariante, un modelo de análisis factorial y un sistema de ecuaciones estructurales, con el cual se validan hipótesis de la relación positiva entre $\mathrm{OE}$ y EC, avanzando en los pocos análisis que se han realizado hasta el momento, y planteada más como reflexión o proposiciones teóricas no validadas. Este es uno de los avances de este estudio; además, se confrontan estadísticamente la relación de complementariedad entre los dos fenómenos, al incluirse cuatro capacidades organizacionales observables que se integran en una variable latente, que actúa como variable mediadora. 
Otros estudios previos han planteado relaciones de interacción (mediación y/o moderación) de determinantes de la OE o del EC, pero no en un análisis que los incluya a ambos. Por ejemplo, Rosenbusch, Rauch y Bausch (2013), han identificado desde un meta análisis, múltiples variables moderadoras y/o mediadoras, entre ellas el aprendizaje, el conocimiento. A su vez, Martins, Rialp, Rialp y Rocio (2015) usaron la OE y el tamaño de empresa demostrando efectos moderadores en el crecimiento de las mismas. Rezaei y Ortt (2018), concluyen que la $\mathrm{OE}$ tiene un efecto moderador significativo en variables de desempeño empresarial.

Como unidad de análisis se observan 137 PYMEs colombianas del sector manufacturero, que han demostrado, debilidades en estructuras organizacionales, procesos de emprendimiento e innovación, todos necesarios para afrontar los retos de sostenibilidad, crecimiento, competitividad e inserción en la economía global.

El trabajo inicia presentando el marco teórico y estudios previos, ampliando diferencias entre OE considerado como intención y el EC como acciones y su relación de complementariedad; seguidamente se presentan y explican las capacidades organizacionales que dinamiza el EC. A continuación, se presenta la metodología; continúa con el análisis de resultados del modelo estadístico, los cuales validan las hipótesis de las relaciones positivas de la $\mathrm{OE}$, tanto con las acciones de EC en que se manifiesta el fenómeno, como con las capacidades analizadas. Finalmente, se presentan las discusiones y conclusiones.

\section{Estado del arte}

Los fundamentos teóricos que se recorren a continuación, permiten explicar la relación de complementariedad entre la OE y el EC, ampliando la comprensión de la forma en que se canalizan la intención y voluntad de emprender, en acciones y proyectos innovadores. Con predisposición a emprender, estrategias, estructuración y asignación de recursos y el desarrollo de capacidades organizacionales adecuadas, se logran comportamientos que impulsan la renovación e innovación empresarial (Ireland, Covin y Kuratko, 2009; Covin y Lumpkin, 2011).

\subsection{OE y EC, aclarando sus significados y la relación de complementariedad}

Puede ocurrir que en una empresa existan atributos de una cultura a emprender, pero el emprendimiento no se hace efectivo, por condiciones socioeconómicas, legales, restricción de recursos y capacidad de ubicarlos, capacidad de ejecución, entre otras razones. Covin y Slevin (1991) establecen que la postura emprendedora y las acciones emprendedoras se han tratado como sinónimos; explican que puede darse el caso de un individuo con perfil psicológico de emprendedor, que no necesariamente actúa como tal; concluyen que la OE puede ser un predictor del EC, pero no siempre corresponde al logro de acciones emprendedoras. Covin y Lumpkin (2011), dos décadas después, ratifican esta diferencia y avanzar en la argumentación. Pasando al plano organizacional la discusión, Covin y Wales (2019) establecen que lo que es verdadero o característico del individuo puede o no serlo para las organizaciones en las que interactúa o se desempeña; una empresa que tiene individuos con espíritu emprendedor no necesariamente es un indicativo de una alta OE. Tampoco lo es el hecho de realizarse acciones de EC y señalan además que hay organizaciones que exhiben altos niveles de EO, pero según los resultados, no logran ser innovadoras y concluyen que las evidencias del espíritu emprendedor no son igualmente generalizadas a lo largo de las empresas, como lo ha apuntado Wales (2016).

La OE puede ser entendida en una doble perspectiva: en primer lugar y acorde a la Teoría del comportamiento planificado -TCP (Bratman, 1987; Ajzen, 1991), es un antecedente del EC, al configurarse como intención, voluntad, mentalidad; en segundo lugar y según la Teoría RBV, es una capacidad organizacional que impulsa las acciones emprendedoras, al estar en una interrelación permanente, como lo explican Lumpkin y Dess (1996), apreciándose la complementariedad entre ambos. 
Covin \& Lumpkin (2011) y Titus Jr, Parker y Covin (2019) coinciden en que el fenómeno de la OE es un atributo organizacional e indicativo de una mentalidad emprendedora por el cual los tomadores de decisiones estratégicas están atentos a oportunidades y seleccionan nuevas ideas de negocios, las cuales evalúan y explotan de una manera planeada, para lograr ventajas competitivas.

La TCP (Bratman, 1987; Ajzen, 1991) se basa en la Teoría de la Acción Razonada (Theory of reasoned action -TRA), la cual se explica en un modelo de relaciones entre actitudes, convicciones, presión social, intenciones y conducta. En la Figura 1 se representa el modelo teórico de Ajzen (1991).

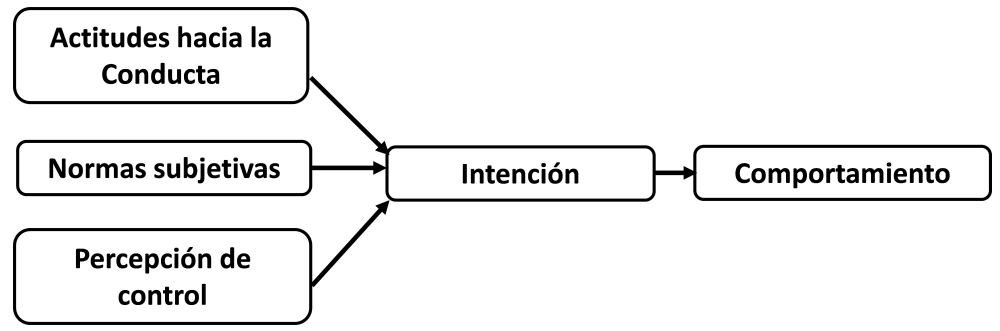

Figura 1. Modelo teórico de Ajzen (1991)

Fuente: Ajzen, I. (1991).

Analizando el fenómeno de creación de empresas, Gelderen, Kautonen y Fink (2015) explican que una intención de comportamiento representa la motivación de una persona para realizar un comportamiento, abarca tanto la dirección (hacer X frente a no hacer X) como la intensidad (cuánto tiempo y esfuerzo está dispuesta a invertir en hacer X). La TCP explica la influencia en la intención de llevar a cabo una conducta, cuando se analizan los recursos y posibilidades, y la presencia de factores que pueden dar campo a la realización distintas acciones.

Esta teoría permite predecir comportamientos deliberados y partir de la base de que pueden ser planeados, además de entender que a una mayor intención, mayor probabilidad habrá de ser realizados. En una aplicación de la TCP en el campo de la gestión empresarial, emprender es un proceso se involucran la planeación, al partir de definir una visión y la capacidad de desplegar esfuerzos y recursos para el logro de objetivos; la influencia de la valoración percibida, el entorno por parte del emprendedor y las normas subjetivas, con valoración de beneficios a lograr, enfocan la actitud hacia los comportamientos (Bratman, 1987; Vélez, 2012).

La intención emprendedora ha sido considerada como un aspecto previo y determinante en los procesos de renovación y desempeño de los comportamientos emprendedores (Mitchell et al., 2007). El Banco Mundial (2015b), en el informe denominado Mente, Sociedad y Conducta, advierte que las intervenciones de política económica y estrategia empresarial deben tener en cuenta los factores psicológicos y sociales específicos que guían la toma de decisiones en un entorno en particular; de así hacerlo, se contribuye a disminuir la brecha entre las intenciones y acciones. El GEM España (2014) señala que el espíritu emprendedor es la capacidad para transformar las ideas en actos, lo que es válido para creación de empresas y para el EC de quienes en éstas se desempeñan.

El análisis del entorno e identificación de nuevas oportunidades, tanto a nivel personal como organizacional, no siempre conduce a una adecuada formulación de planes y proyectos y al compromiso de asignación de recursos para ejecutarlos. Existe una brecha entre las intenciones de emprender y las acciones (Schlaegel y Koenig, 2014; Gelderen, Kautonen y Fink, 2015), por lo que es importante seguir analizando y proponiendo modelos que ayuden a cerrarla. Esto puede ocurrir por las razones antes mencionadas, además falta de ambición, débil capacidad de ejecución, cambio de condiciones económicas y coyunturales, tipo de administración de las empresas, entre otros (Titus Jr, Parker y Covin, 2019). 


\subsubsection{La orientación emprendedora como voluntad}

Mcfadzean, O'Loughlin y Shaw (2005) explican que al no existir actitudes emprendedoras que permitan explotar las oportunidades que se le presentan a las empresas, la innovación es más aspiracional y no llega a concretarse. Los autores señalan la importancia de la existencia de personas y organizaciones con el deseo y la voluntad o predisposición de emprender e innovar. La visión emprendedora es referida a lo que la empresa quiere lograr en el futuro, pero las acciones emprendedoras incluyen interacciones, asignación de recursos, desarrollo de nuevas ideas, explotar las oportunidades potenciales identificadas.

La OE se constituye en una actitud o conducta fundamental para lograr el EC, manifestada en el espíritu emprendedor o mentalidad emprendedora de fundadores y empleados de las organizaciones. Después de dos décadas de analizar el constructo OE, Covin y Wales (2011) señalan que la postura emprendedora es el elemento central de los procesos emprendedores permite a las empresas su flexibilidad estratégica y rápida capacidad de adaptación a un entorno en constante cambio, aprovechando el conocimiento (Wiklund y Shepherd, 2003) y la información para identificar y explotar oportunidades (Zahra y George, 2002).

\subsubsection{El emprendimiento corporativo como acciones}

Tradicionalmente, el CE se ha relacionado con acciones (Zahra, 1993; Antoncic y Hisrich, 2001;), siendo coherente con la Teoría de la Destrucción creativa (Schumpeter, 1942), referida a la innovación, desarrollo de nuevos productos o mejora de los existentes, de los procesos y tecnologías o incursionando en nuevos mercados. Kuratko y Audretsch (2009) enfatizan en que las acciones emprendedoras son el conducto a través del cual se practica el emprendimiento en las organizaciones.

Recientes trabajos apuntan a determinar el impacto de los procesos de emprendimiento con variables explicadas o de resultado, usando el constructo Innovative Performance (Goodale, Kuratko, Hornsby y Covin, 2011; Hasan, Syyedhamzeh y Ali, 2013), el cual prevalece en el concepto de destrucción creativa de Schumpeter (1942) y en la definición de innovación que da la OCDE -Oslo (2005) ya presentada anteriormente. Por su parte, la OCDE (2012) asocia el emprendimiento a la actividad empresarial, entendida como acciones relacionadas con la identificación y explotación de nuevos productos, procesos o mercados.

Aclarando la diferencia entre los significados de la OE como intención- voluntad y el EC como acciones, en este trabajo se considera que el EC se refleja en acciones y por lo tanto se empleará esta perspectiva ya reconocida por distintos autores para analizar el efecto de la OE y de las capacidades en el EC eficaz (Covin y Lumpkin, 2011; Mcfadzean, O’Loughlin y Shaw, 2005; Hasan, Syyedhamzeh y Ali, 2013).

Rauch, Wiklund, Lumpkin y Frese (2009) subrayan que la actitud innovadora es la predisposición a participar en la creatividad y la experimentación, para la introducción de nuevos productos, servicios o tecnologías, a través de la I + D. Se observa que en ambas definiciones se relacionan estrechamente con la intención, estando presente la voluntad, entendida como deseo, predisposición o inclinación que conduce a la acción, lo que permite establecer la siguiente hipótesis a validar, para establecer la relación positiva entre los fenómenos de la OE y el EC.

Hipótesis 1: La orientación emprendedora impacta positivamente en la implementación de acciones de emprendimiento corporativo.

Esta hipótesis recoge la proposición teórica planteada por Bouchard y Basso (2011), y las reflexiones señaladas en el estado del arte de esta propuesta, tanto de autores seminales, como de otros más recientes con similar interés (Schlaegel y Koenig, 2014; Todorovic, Todorovic y Ma, 2015; Gelderen, Kautonen y Fink, 2015), pero enfocados en el campo de la creación de empresas y no en del emprendimiento de las organizaciones, lo que representa novedad en el estudio del EC y de la OE como campo de perspectiva. 


\subsection{Recursos y capacidades que impulsan el emprendimiento corporativo}

Acorde a la Teoría del comportamiento Planificado (Bratman, 1987; Ajzen, 1991; Vélez, 2012), desde la identificación y concepción de los proyectos hasta su puesta en marcha, se incorporan procesos de planeación interdisciplinarios. Sapag, Sapag, Sapag (2014) incorporan distintas áreas funcionales de la organización que se involucran e interrelacionan en la preparación y evaluación de proyectos de inversión.

A continuación, se analizan capacidades que se van desarrollando cuando hay una firme OE y son a la vez antecedentes del EC, explicando en su rol de mediadoras de la relación de complementariedad entre la $\mathrm{OE}$ y el EC. Algunas de las que se presentan, han sido más estudiadas que otras en la relación con los fenómenos, estudiados generalmente por separado. Para que una organización emprenda, debe disponer de recursos heterogéneos y cumplir con las propiedades de ser valiosos, escasos y difíciles de imitar o de sustituir, criterios que se relacionan con el desarrollo y la sostenibilidad de las ventajas competitivas y que se amplían adelante (Wernerfelt, 1984; Barney, 1991; Teece, Pisano y Schuen, 1997). La importancia de los recursos y capacidades se hace mayor cuando se integran de una manera coordinada, flexible y eficiente para aventajar a la competencia.

La definición de innovación OSLO (2005) incluye iniciativas de tipo gerencial o nuevos modelos administrativos, modificación de estructuras organizacionales, desarrollo de habilidades -procesos - técnicas de gestión, capacidades, implementados para aumentar la competitividad, el crecimiento, desempeño y el valor de las compañías (Birkinshaw, Hamel, y Mol, 2008). Las innovaciones gerenciales hacen parte de las capacidades más complejas y difíciles de imitar, adquirir y copiar una vez que son desarrolladas, características explicadas por Barney (1990). Dichas capacidades son objeto de nuevos campos de investigaciones que explican el EC innovador y representan avances sobre el enfoque tradicional de las innovaciones tecnológicos (Birkinshaw, Hamel, y Mol, 2008; Zhang, Khan, Lee y Salik, 2019). Por lo multidimensional del tema, se analizan simultáneamente cuatro de estas capacidades, de alto impacto en la relación de complementariedad entre la OE y el EC.

\subsubsection{Absorción del conocimiento}

El aprendizaje colectivo, junto con la capacidad de absorción del conocimiento, tienen incidencia en el EC de la empresa, al conducir a individuos al desarrollo de conductas emprendedoras y diferentes estilos en toma de decisiones, métodos o prácticas administrativas, e identificar y explotar proactivamente oportunidades del entorno (Nonaka y Takeuchi, 1995; Chetty y Wilson, 2003; Wiklund y Shepherd, 2003; Taheri, Bititci, Gannon y Cordina, 2019). La aplicación y creación del conocimiento, representa importancia estratégica para la empresa (Grant, 1996); el aprendizaje organizacional, la gestión de la tecnología y las capacidades cognitivas gerenciales han fundamentado distintas líneas tradiciones de investigación. Para Grant, el conocimiento, ofrece una base teórica para la comprensión de los retos de renovación e innovación de las organizaciones, por lo que presenta diversas características para que el conocimiento se convierta en una capacidad especial, permitiendo su transferencia, agregación, apropiación, especialización, coordinación y cooperación.

Zahra y George (2002) presentan un modelo que integra las dos etapas más importantes para el proceso de convertir el conocimiento en las acciones que producen una ventaja competitiva: en primer lugar, está la capacidad de absorción, que incluye la adquisición y asimilación del mismo; segundo, la capacidad de absorción realizada, que incluye aplicar el nuevo conocimiento para la transformación organizacional y la explotación de oportunidades. Chetty y Wilson (2003) establecen que buena parte del conocimiento en las empresas es generada cuando sus miembros se comprometen en relaciones, comparten información y trabajan colaborativamente.

Sapienza, De Clercq y Sandberg (2005) establecen que el aprendizaje es un proceso en el que se encaminan 
las firmas para desarrollar conocimientos; la atención que presta una empresa a analizar información del entorno se relaciona con la manera como desarrolla su capacidad de aprendizaje, convirtiéndose en un recurso cada vez más crítico y valioso para la economía basada en el conocimiento. Por esta razón, sostienen los autores que aprendizaje y creación de conocimiento son las actividades estratégicas más importantes de las firmas encaminadas a crear ventajas competitivas sostenibles.

Taheri, Bititci, Gannon y Cordina (2019) desarrollan medidas específicas del fenómeno del desarrollo del aprendizaje y el conocimiento mediante el análisis de información proveniente de distintas fuentes, para que los empleados de la firma logren una mayor percepción y comprensión de los cambios turbulentos del mercado, proveedores, competidores, clientes, regulaciones, entre otros y realicen acciones de emprendimiento innovador como respuestas a sus interpretaciones.

\subsubsection{Capacidad de financiación}

Los emprendimientos requieren recursos de distintos tipos, entre los cuales están los financieros. Las teorías financieras soportan el análisis de las inversiones, las fuentes con que se financian y la estructura de capital que incide en la determinación de las tasas mínimas requeridas de retorno que se exigen a las iniciativas que se analizan e integran el presupuesto de capital de la compañía. La capacidad financiera que se explorada en el estudio, considerada un factor fundamental para la implementación de emprendimientos corporativos (Stevenson y Jarillo, 1990). Aunque desde el campo de las finanzas es clara su importancia e impactos, como antecedentes de los dos fenómenos que se estudian en este trabajo, es poco lo que se ha analizado o se le da de hecho, cuando se analizan la OE y el EC, cuando es de reconocida aceptación que el acceso a fuentes adecuadas de financiación puede impulsar las inversiones que se requieren en cualquier emprendimiento, pero el no tenerlo, es una de las razones que explica la brecha entre los constructos.

Las inversiones necesarias para realizar emprendimientos son estimadas en los presupuestos de capital de la firma, en función de la creación de valor esperada, rentabilidad estimada y coherencia con los objetivos estratégicos de la organización, como ha sido establecido por diversos trabajos y por ello, el acceso a diversas fuentes de financiación, internas y externas a la empresa, es determinante de las posibilidades de implementar proyectos de inversión (Stevenson y Jarillo, 1990; Zahra, 1993; Wiklund y Shepherd, 2003).

Para las PYMEs, el acceso al financiamiento es uno de los factores que más preocupa a sus administradores, así como el costo de los créditos, las reciprocidades y las exigentes garantías por las entidades crediticias. La selección y uso de diferentes fuentes de financiación son aspectos que estas organizaciones deben considerar a partir de un proceso integrado de planeación financiera y proyección de estados financieros, de tal manera que se pueda establecer una estructura financiera más adecuada (Zahra, 1993; Wiklund y Shepherd, 2003; Brealey, Myers y Allen, 2006).

Las decisiones de financiación requieren de una clara comprensión del impacto en el costo de capital y en el valor de la compañía, así como de la definición de la estructura o participación deseada entre fuentes propias o de terceros; entre las primeras, está la generación interna de fondos, provenientes de las utilidades no repartidas, de las depreciaciones y amortizaciones (por ser estos gastos no desembolsables en efectivo), o de nuevos aportes de capital de los dueños.

En las segundas, como parte de fuentes de terceros, están los pasivos a seleccionar según las opciones existentes en el mercado, las disponibles en instituciones financieras, apoyos del Estado por disposiciones de políticas económicas que apoyan la innovación y el impulso de determinados sectores, el buen manejo financiero de la empresa, la forma en que ha honrado sus compromisos de pago en el pasado, y además en función de los costos, plazos, condiciones, garantías y lo que es fundamental, de conocer de la existencia de las mismas y de entender las conveniencias o desventajas de cada una (Brealey, Myers y Allen, 2006). 


\subsubsection{Redes externas de cooperación}

Una capacidad organizacional en la cual cada día más empresas se comprometen con su desarrollo, es el trabajo en redes externas de cooperación y configuración de capital social. Las redes sociales han sido definidas como un conjunto de actores sociales que pueden ser individuos u organizaciones con una serie de vínculos entre ellos (Hoang y Antoncic, 2003). Crecen en interés los estudios que explican las redes externas como uno de los factores que inciden en el desarrollo organizacional y en el EC o la OE (Gulati, 1998; Hoang y Antoncic, 2003). Martins, Rialp, Rialp y Rocio (2015), señalan que aquellas empresas cuyos fundadores y directivos participan activamente en redes externas de colaboración, desarrollan capacidades cognitivas que les permiten estar atento a los cambios del entorno, reconocer nuevas oportunidades, estructurar recursos y capacidades de forma sobresaliente y crear productos superiores a los del mercado.

Participando activamente de redes específicas, las empresas establecen contacto permanente e interrelaciones con otros agentes, que les provee de un lado información formal o informal, la cual pueden volverse conocimiento y aplicarse en el desarrollo de aprendizajes que les permita identificar oportunidades de inversión y desarrollar emprendimientos corporativos (Zahra y George, 2002).

La interacción de experiencias propias o las desarrolladas por proveedores, clientes y por otras organizaciones en el ámbito nacional permiten obtener información que puede ser procesada para desarrollar conocimientos, investigar, lanzar nuevos productos o servicios, desarrollar nuevas capacidades, mejoras en los procesos y diversos proyectos e incluso implementarlos conjuntamente con estrategias de alianzas y joint venture o inversiones conjuntas. En el contexto internacional, también se dan las redes que buscan aprovechar marcos de cooperación, tratados comerciales e incentivos a la inversión extranjera.

\subsubsection{Formulación, evaluación y gestión de proyectos}

Como cuarta y última capacidad, se exploran y confirman rutinas que hacen parte de la gestión administrativa, relacionadas con procesos de formulación, evaluación financiera y gestión de proyectos. Pese a estar relacionada con los procesos de emprendimiento y representar una gran relevancia para el EC eficaz, no se encontró en la revisión de literatura que se haya analizado en relación a los dos fenómenos estudiados, así sea por separado y menos aún contrastada cuantitativamente. La relevancia de la capacidad de formular, evaluar y gestionar los proyectos como determinante del EC, radica en que se relaciona directamente con los procesos de emprendimiento, señalados en la Teoría del Comportamiento Planificado-TCP (Bratman, 1987; Vélez, 2012), integrados en la planeación estratégica y la ejecución de programas y proyectos concebidos (Rosillo, 2008; Arboleda, 2013).

Esta capacidad entra en acción después que han sido reconocidas oportunidades, resultado del estar alertas a los cambios que se presentan en el entorno, e interpretar los efectos que tienen los mismos para la compañía en términos de amenazas y oportunidades, generándose ideas de proyectos innovadores (Kirzner, 1973; Zarha, 1993); se integra por los procesos de formulación y evaluación de proyectos desde distintas perspectivas disciplinares, presupuestos de capital, proyecciones financieras, estimación de criterios y acorde a éstos, la aceptación o rechazo de los proyectos de inversión; en una etapa posterior inicia la implementación y gestión de los proyectos aprobados, el control durante la etapa de ejecución e incluso, seguimiento posterior durante la operación, para verificar el cumplimiento de los objetivos perseguidos con la iniciativa desarrollada (Sapag, Sapag, Sapag, 2014; Brealey, Myers y Allen, 2006).

Adicionalmente, ante la cantidad de oportunidades de inversión y recursos limitados, se hace necesario optimizar los procesos de gestión administrativa y asignación de recursos de inversión. La tarea de selección de proyectos a desarrollar entre múltiples alternativas de inversión y con limitación de recursos, es una rutina fundamental relacionada con esta competencia, que incorpora procesos de priorización y jerarquización de 
los proyectos innovadores (Cardona, Cardona, y Naranjo, 2012). Adicionalmente, una buena dirección de proyectos que conlleve a las organizaciones a desarrollar proyectos con éxito y con los recursos, tiempo y dinero presupuestados, también son procesos fundamentales para el EC eficaz. Cardona, Martins y Velásquez, 2017) aportan a su definición:

La Capacidad de evaluar y gestionar proyectos son procesos que impulsan persistentemente el emprendimiento corporativo, los cuales incluyen la concepción de una idea, la formulación de proyectos, el análisis de viabilidad, las decisiones de inversión e implementación hasta su puesta en marcha, bajo adecuada gestión y control, e interactúan con competencias blandas de liderazgo, comunicación y trabajo en equipo para el logro de los objetivos propuestos de una determinada iniciativa (p.50).

Del análisis anterior, es de esperarse que cuando hay una firme OE, en las organizaciones se vayan desarrollando con el tiempo mejores capacidades de EC, por lo que se estructura la siguiente hipótesis a ser validada:

Hipótesis 2: La orientación emprendedora impacta positivamente en la estructuración de capacidades de emprendimiento corporativo.

\subsection{Emprendimiento corporativo materializado en acciones}

Diversos estudios emplean variables que permiten analizar y/o medir las consecuencias de los procesos de EC, manifestado en realizaciones y acciones concretas (Mcfadzean, O'Loughlin y Shaw, 2005; Goodale, Kuratko, Hornsby y Covin, 2011; Hasan, Syyedhamzeh y Ali, 2013). El Parlamento Europeo (citado en GEM España, 2014, p.125), se refiere a competencias emprendedoras y el espíritu de la iniciativa de las empresas, como "la habilidad de la persona para transformar las ideas en actos". Kuratko y Audretsch (2009) resaltan la existencia de factores que inciden en las acciones emprendedoras para la implementación de la estrategia corporativa dirigida hacia el EC; señalan que para que las acciones emprendedoras individuales y en equipo florezcan, lo más importante es la habilidad de establecer una visión desde la alta dirección, dar soporte administrativo, organizar las personas, tareas y proveer los recursos suficientes que las financien e incluyen acciones de creación, renovación o innovación que se producen dentro o fuera de una organización; para los autores, las acciones emprendedoras son vistas como la ruta crítica hacia la ventaja competitiva y mejora del desempeño en el Siglo XXI, "son el conducto a través del cual se pone en práctica el emprendimiento en las organizaciones" (p.60),

En este estudio se aprovecha los espacios aún abiertos para la investigación sobre las relaciones y la complementariedad por la operatividad de OE y EC, para contribuir a la teoría y posibilitar la mejora en la efectividad del emprendimiento, materializado en acciones que encierran incertidumbre en los resultados esperados (Ireland, Kuratko y Morris, 2006; Kuratko y Audretsch, 2009; Miller, 2011; Covin y Lumpkin, 2011; Covin y Wales, 2019;).

Sintetizando sobre las relaciones entre las categorías y variables provenientes del marco teórico, se espera que una empresa logre ventajas competitivas sostenibles, al estructurar diversas capacidades e implementar acciones o proyectos innovadores bajo incertidumbre (Mintzberg, 1994; Grant, 2006; Miller, 2011; Covin y Lumpkin, 2011).

\subsection{OE, las capacidades y acciones de EC: efectos directos e indirectos}

Es de esperarse, acorde a las teorías de la estrategia, que una empresa que pretende impulsar la conducta emprendedora e implementar acciones de EC, debe ajustar sus capacidades para alcanzar los objetivos 
estratégicos y ventajas competitivas, lo que sintetiza como la OE de una empresa como voluntad y actitud, se concreta en acciones emprendedoras, complementándose entre si ambos fenómenos: Se considera que una empresa con alta Orientación emprendedora, cuando se caracteriza por la proactividad, asunción de riesgos y carácter innovador, reúne atributos por parte de sus directivos y talento humano, como la intención y la persistente voluntad de emprender, el permanecer alertas a oportunidades del entorno, analizarlas en función de su viabilidad, elegir entre múltiples opciones de inversión, asignar recursos y realizar constantemente acciones de Emprendimiento corporativo.

Del marco teórico, para analizar la complementariedad de la OE y el EC, se establecen hipótesis adicionales que se validan estadísticamente, y se presenta el modelo teórico que las integra: Hipótesis 3: La estructuración de capacidades de emprendimiento corporativo en las PYME impacta positivamente en la implementación de acciones de emprendimiento corporativo.

Hipótesis 4: La incidencia positiva entre orientación emprendedora y las acciones de emprendimiento corporativo de las PYME, se modifica cuando es intermediada por las capacidades de emprendimiento corporativo.

Las relaciones entre $\mathrm{OE}$ y las capacidades que se desarrollan para gestionar los procesos de emprendimiento de manera más efectiva, y que interactúan como determinantes del EC, se plasman en el modelo teórico estructural que se observa en la Figura 2:

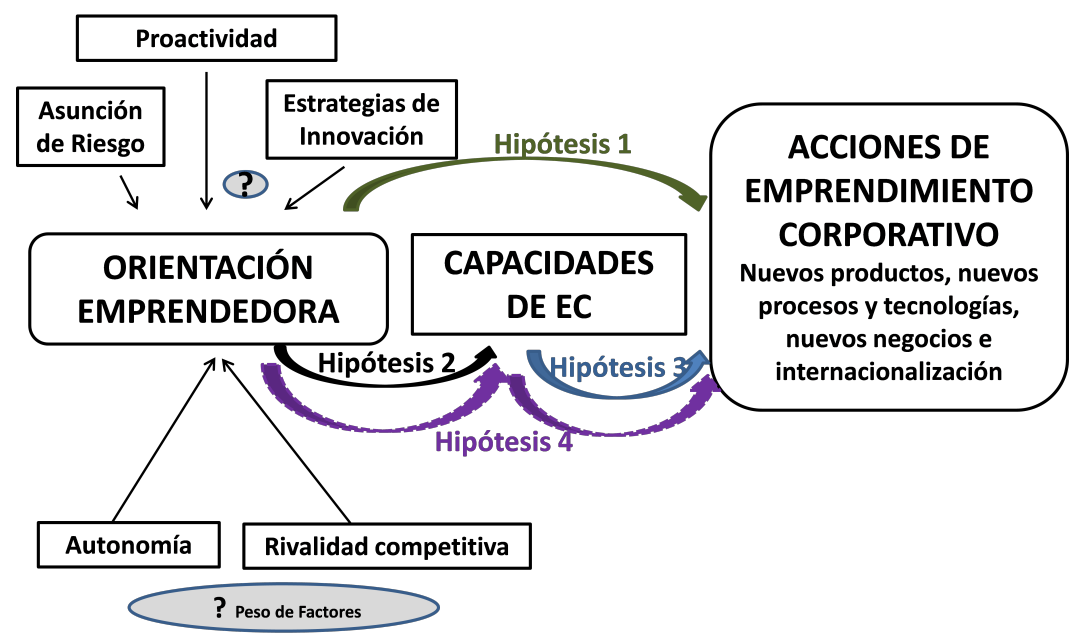

Figura 2. Orientación emprendedora y capacidades para el emprendimiento corporativo en las PYME: de la intención a la acción.

Fuente: Elaboración propia

\section{Metodología}

La metodología utilizada se fundamenta en procedimientos del análisis multivariado entre los cuales se aplica el análisis factorial exploratorio y el confirmatorio; el planteamiento teórico de la propuesta se recoge utilizando sistemas de ecuaciones.

Se analiza la OE en las PYME, analizando cinco dimensiones en forma conjunta, las cuales se validan en el modelo desarrollado, incluyéndose las tres establecidas por Covin y Slevin (1991), dándole una connotación a la innovación como estrategia y no como acciones; además se incorporan las dos dimensiones que complementaron Lumpkin y Dess (1996).

La base de datos utilizada para la estimación de los modelos estadísticos propuestos, se estructuró con la información proveniente del trabajo de campo, que contó con 137 PYMEs manufactureras, de una base de 
un total de 543, que cumplieron con los criterios definidos de elección, tener más de 3.5 años de establecidas y tener entre 10 y 200 empleados (clasificación PYMEs, según ley 590 del 2.000).

Se seleccionan este tipo de empresas por representar una alta parte de la población empresarial y ser generadoras de la mayor cantidad de empleos, a riesgo de no tener la información ideal para construir los datos con los que se estiman los modelos multivariables para probar las hipótesis propuestas, lo que puede ocurrir por las debilidades de gestión de que les son conocidas en función a su tamaño, y dadas las restricciones de recursos humanos y financieros. A diferencia, en grandes empresas, existen estructuras organizacionales y procesos de gestión son más definidos y especializados, que pueden arrojar resultados distintos y más posibilidad de confirmar las hipótesis.

De las empresas que se les invitó a participar, se logró una tasa efectiva de respuesta de 25.23 por ciento, lo que representa una buena proporción, acorde a estudios empíricos previos; las PYMEs manufactureras analizadas se localizan en el Área Metropolitana de Medellín, reconocida por su tradición emprendedora. El muestreo se realizó por conveniencia, tratándose de una participación voluntaria en la disponibilidad de las empresas para colaborar con la investigación, dada la dificultad de realizar un sistema de muestreo probabilístico, pero asegurando que cumplieran con los parámetros de selección: tener como mínimo 3.5 años de establecidas, teniendo organizaciones desde 4 años de creadas hasta 112 años, y en promedio 23 años de existencia legal. Además, las unidades encuestadas, debían ser pequeñas y medianas empresas, registrándose empresas desde 12 empleados hasta 250, con un promedio de 75 empleados.

A los datos que se usan para construir el modelo, se le aplican pruebas para establecer la no existencia de sesgo de no respuesta sin encontrar diferencias sustanciales entre las empresas que decidieron participar en la realización del estudio- que conformaron la muestra, con aquellas que no la diligenciaron. Se verificó la no presencia del sesgo de método común, evitando el error de medida que distorsione las conclusiones de la investigación.

Se establecieron dos pasos para garantizar que no haya una presencia del sesgo del método común, lo que generaría un error de medida que distorsione las conclusiones de la investigación; inicialmente siguiendo la metodología desarrollada por Podsakoff, MacKenzie, Lee y Podsakoff (2003), para reducir en cierta medida la subjetividad con que responden los encuestados, se dejó claro a la hora de responder la encuesta que se protegería el anonimato de la compañía y de quien la diligencia, respondida por el gerente de la empresa o en su defecto un alto directivo.

Segundo, bajo el supuesto que si una proporción alta de la varianza del método común se encuentra presente, entonces un solo factor resultará significativo en el análisis factorial, (Podsakoff, MacKenzie, Lee y Podsakoff, 2003; Meade, Watson y Kroustalis, 2007); para ello se realizó un análisis factorial exploratorio de todos los ítems de los constructos, en este caso para OE, capacidades de emprendimiento corporativo y Acciones de EC como si fueran uno solo y se comprobó que no existiera un solo factor que explique la mayor proporción de la varianza.

El cuestionario se estructura con respuestas a las preguntas en escala Likert; para encontrar las variables a analizar en el estudio, la encuesta se estructuró con 55 variables observables y que posteriormente integran las tres variables latentes, validadas en el análisis factorial: OE, que comprende 26 preguntas; las capacidades, denominadas Recursos y Capacidades -RECCAP, que incluye 22 preguntas; por último, las acciones de emprendimiento en que se concreta el EC, con 7 preguntas.

Para el modelo, se validan las dimensiones que integran la OE, y se establecen RECCAP y EC, resultando tres factores a los cuales se les realiza análisis factorial exploratorio y se comprueba la validez en el planteamiento del modelo; los dos fenómenos estudiados simultáneamente OE y EC, se analizan en una relación no explorada hasta el momento y tampoco confrontada cuantitativamente en estudios previos, por considerarse como sinónimos (Antoncic y Hisrich, 2001) o porque han sido usados indistintamente (Lumpkin y Dess, 1996). 
REMEF (The Mexican Journal of Economics and Finance)

En este trabajo, estos factores son mediados por las Capacidades de Emprendimiento Corporativo, como se observa en las figuras 2 y 3 .

En la fase inicial, se realizó un análisis factorial exploratorio- EFA, se halló la matriz de correlaciones de las variables, se obtienen los factores, se retienen aquellos con valor propio mayor a 1 y se hallan los resultados correspondientes a la representación, bajo una rotación ortogonal varimax propuesto por Kaiser (1958), que permite descomponer la varianza total entre los distintos factores e identificar cuáles variables integran cada factor.

Después se validan las hipótesis propuestas por medio de sistemas de ecuaciones estructurales-SEM. Las estimaciones se realizaron considerando el método de máxima verosimilitud, que de acuerdo con Pérez (2008) es no sesgado, eficiente e invariable al tipo de escalas, y recomendado cuando el tamaño de la muestra se encuentra entre 100 y 200 observaciones; finalmente se interpretan los efectos directos y mediados de las variables observadas y latentes, y se realizan las pruebas de bondad de ajuste, consideradas pertinentes.

\section{Resultado y discusión}

Una breve síntesis de estadísticas descriptivas de cada variable y de los factores encontrados, dan cuenta de que el $54,7 \%$ de las empresas estudiadas presentaron calificaciones por debajo de 3 en su orientación emprendedora, siendo la proactividad la de resultado más bajo. De la misma manera, el 49,6\% obtuvo calificaciones por debajo de 3 en el factor capacidades de emprendimiento corporativo, siendo la más baja la absorción de conocimiento, seguida de la capacidad de evaluar y gestionar proyectos.

Específicamente, en las funciones relativas a la capacidad de evaluar y gestionar proyectos para las PYME analizadas, se evidenciaron debilidades en metodologías de flujos de caja y criterios de decisión usados y en el seguimiento y control a los proyectos implementados; además existen falencias más fuertes en cuanto a las metodologías para priorizar entre los múltiples proyectos, resultantes de una mayor orientación emprendedora y procesos de emprendimiento corporativo más efectivos. Debilidades también se encontraron en la conformación de redes externas, así como los procesos de absorción de conocimiento, resultados coherentes con estudios previos realizados para el contexto colombiano, que han determinado que este tipo de empresas, adolece de la estructuración de áreas funcionales que permitan procesos de planeación eficaces y de evaluación y gestión de proyectos (Barriga, 1998; Cardona, 2012). En el Anexo 1, Estadística descriptiva por variables de las distintas dimensiones, se observan los porcentajes de las PYMEs con buena calificación en los constructos analizados.

Del análisis multivariante, en la fase factorial exploratoria y para cada constructo, se consideran el índice Kaiser-Meyer-Olkin - KMO y el test de esfericidad de Bartlett $(\mathrm{p}<0.01)$ con el fin de identificar la pertinencia de realizar un análisis exploratorio y si la integración de los factores se hace de forma eficiente. El test KMO es una medida de adecuación de la muestra desarrollado por Kaiser (1974) y relaciona los coeficientes de correlación observados entre las variables analizadas, resultando los coeficientes de correlación parcial entre las variables. Cuanto más cerca de 1 tenga el valor obtenido del test KMO, implica que la relación entre las variables es alta.

Alpha de Cronbach: Desarrollado por Cronbach (1951), una medida de consistencia que evalúa la consistencia del modelo en su totalidad así como la de las dimensiones obtenidas (Hair et. al, 1999). Este se mueve entre 0 y 1 , y el criterio generalmente aceptado es que el límite inferior es 0.6 en investigaciones. Se calcula el alpha de Cronbach para cada variable, constructo y dimensión. 


\subsection{Orientación Emprendedora}

Del análisis exploratorio se obtienen cinco factores que explican 94.53 por ciento de la varianza total. $\mathrm{El}$ análisis factorial exploratorio es consistente con las teorías de $\mathrm{OE}$, al validarse las cinco dimensiones que integran el constructo (Covin y Slevin, 1991; Lumpkin y Dess (1996), siendo coherente con trabajos previos (Dess y Lumpkin, 2005; Wiklund y Shepherd, 2003).

Los resultados de la estimación validan el efecto positivo y significativo las dimensiones que integran la $\mathrm{OE}$, de acuerdo a sus coeficientes estimados y el test de esfericidad de Bartlett $(\mathrm{p}<0.01)$ : autonomía $(0.7320)$, asunción del riesgo (0.7680), proactividad (0.7736), voluntad de innovar medido por estrategias de innovación (0.6393) y agresividad competitiva (0.4816).

\subsection{Capacidades de emprendimiento corporativo}

El análisis exploratorio permite obtener como resultado el factor Capacidades de EC- CECO, que resultó significativo para las capacidades analizadas, según el Alpha de Cronbach de 0.8832 y el test de esfericidad de Bartlett: absorción de conocimiento (0.8026), redes externas de cooperación (0.8111), evaluación y gestión de proyectos (0.8666), capacidades de financiación (0.7150).

Después de revisar la literatura sobre EC, no se evidenció la existencia de un constructo teórico que incluya diversas capacidades, analizadas por separado en diversos trabajos; por lo tanto, teniendo en cuenta el análisis de los resultados del modelo factorial usado, se establece el constructo Capacidades de Emprendimiento Corporativo, el cual integra diversas rutinas que impulsan las actividades emprendedoras en las organizaciones, esperando aportar a la literatura y en el campo práctico, le sirva a las empresas que buscan desarrollar un EC innovador, eficaz y sostenible:

Dado que la dimensión Capacidades de EC- CECO no ha sido definida teóricamente como constructo por su composición multidimensional -al integrarse por distintas capacidades que han sido analizadas por separado, y de acuerdo con los resultados del CFA, se sigue la siguiente definición, que aporta a la literatura de la gestión y a las empresas que buscan desarrollar un emprendimiento corporativo eficaz y permanente (Cardona, Martins y Velasquez, 2016, p.16):

\subsection{Acciones de Emprendimiento Corporativo}

La implementación de acciones de EC en los que se concreta el fenómeno, permite obtener estadísticos KMO de 0.573, Alpha de Combrach de 0.6731 y el test de esfericidad de Bartlett. Con las variables consideradas, los resultados dejan ver un factor que explica el 74.65 por ciento de la varianza total, el cual contiene seis variables.

Estos hallazgos son coherentes con los estudios de Zahra (1993), Mcfadzean, O'Loughlin y Shaw (2005) y Hasan, Syyedhamzeh y Ali (2013), quienes presentan escalas para medir los resultados en que se materializa el EC, relacionados con los tipos de iniciativas emprendedoras o resultados de innovación, tomada en sentido amplio; y donde señalan la relación positiva del fenómeno con el desempeño empresarial. Partiendo de la validez del análisis factorial exploratorio realizado previamente, se procede a corroborar las hipótesis planteadas por medio de ecuaciones estructurales, en un modelo conjunto, que se observa en la Figura 3. 


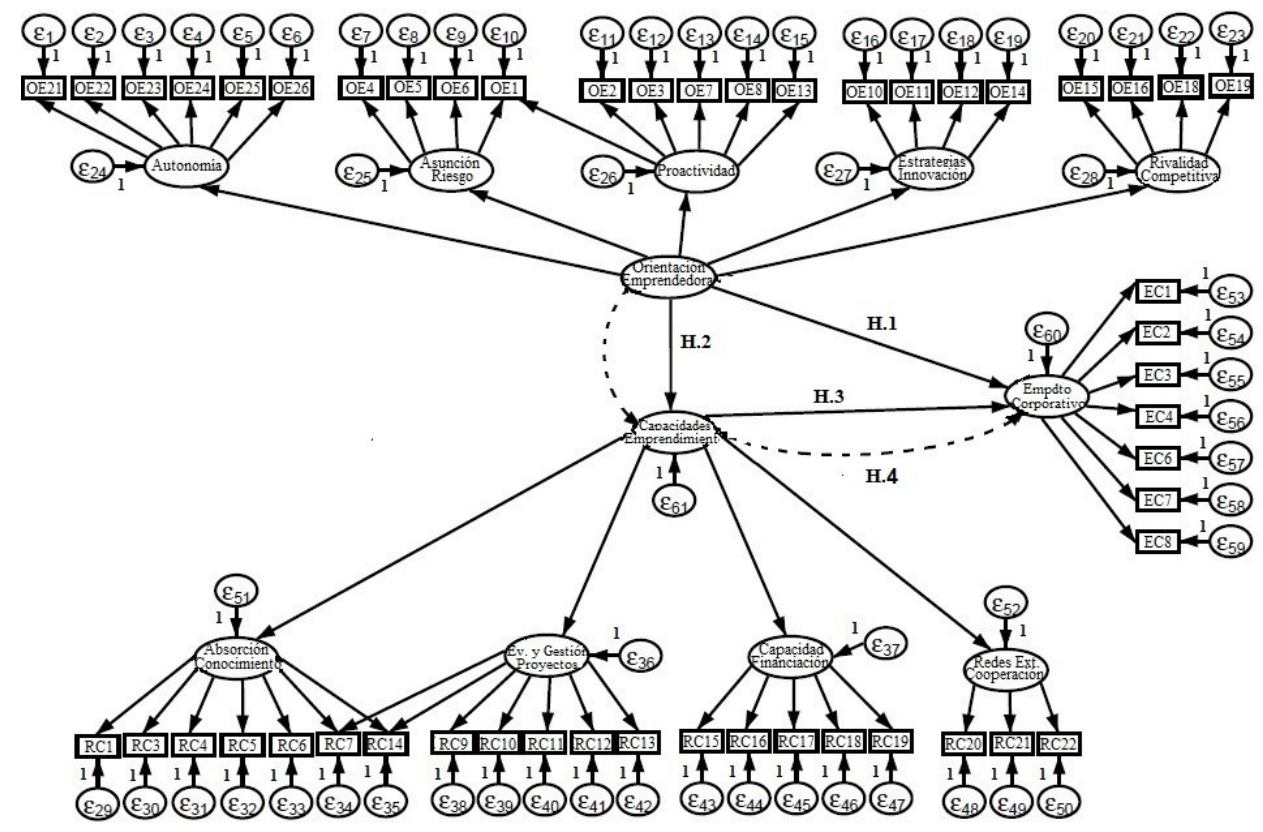

Figura 3. Especificación del modelo para las hipótesis

Fuente: Elaboración propia

A continuación, se presentan en la tabla No. 1, los resultados para la estimación del modelo con las cuatro hipótesis:

Tabla 1. Resultados estimación para las hipótesis

\begin{tabular}{|l|c|}
\hline \multicolumn{1}{|c|}{ Hipótesis } & $\begin{array}{c}\text { Coeficientes } \\
\text { Estimados y } \\
\text { Errores Estándar }\end{array}$ \\
\hline Orientación emprendedora $\rightarrow$ Acciones de Emprendimiento corporativo & $0.749^{* * *}$ \\
\hline Orientación emprendedora $\rightarrow$ Capacidades de emprendimiento corporativo & $(0.147)$ \\
\hline $\begin{array}{l}\text { Capacidades de emprendimiento corporativo } \rightarrow \text { Acciones de } \\
\text { Emprendimiento corporativo }\end{array}$ & $0.888^{* * *}$ \\
\hline $\begin{array}{l}\text { Orientación emprendedora } \rightarrow \text { Capacidades de emprendimiento corporativo } \rightarrow \\
\text { Acciones de Emprendimiento corporativo }\end{array}$ & 0.067 N.C \\
\hline
\end{tabular}

\section{Notas: Coeficientes estimados. ${ }^{* * *} \mathrm{p}<0.001$}

N.C: No confirmada

Fuente: Elaboración propia

De la tabla No.1 se pueden obtener las siguientes conclusiones: se validan las Hipótesis 1 y 2, con efectos directos y positivos de las relaciones contrastadas, las que resultan estadísticamente significativas. Se validan las Hipótesis 2 y 3, con efectos directos y positivos de las relaciones evaluadas, aunque no resulten estadísticamente significativas. La no significancia estadística de las hipótesis tres y cuatro se explica posiblemente, 
porque en las PYME analizadas como unidad muestral de este trabajo, han desarrollado esta capacidad, pero aún con debilidades en los procesos que integran. Es de conocimiento general que estas empresas adolecen de la estructuración de áreas funcionales que permitan eficaces procesos de planeación y de evaluación y gestión de proyectos, lo que se corrobora en estudios anteriores para el contexto colombiano (Barriga, 2006; Cardona, Cardona, y Naranjo, 2012).

Estos resultados pueden ser vistos con mayor claridad en la Figura 3, donde la flecha punteada representa la relación mediada por la estructuración de Capacidades de emprendimiento corporativo, de un efecto directo entre OE sobre el emprendimiento corporativo.

Para el modelo completo se estimaron cuatro pruebas de bondad de ajuste: el Chi-2 ajustado (Chi2 /grados de libertad) obteniendo un valor de 1.847 que al estar por debajo de 3.0 puede interpretarse como un buen ajuste del modelo; igual el índice Root Mean Square Error of Approximation -RMSEA se estimó en 0.079, encontrándose en el rango adecuado; el coeficiente de determinación CD- 0.777, que se asemeja al R2 para modelos de regresión e indica el buen ajuste cuando se aproxima a 1; finalmente el test CFI (0.635) o índice de ajuste comparativo, que se encontró en un rango aceptable.

En la Figura 4, puede apreciarse las estimaciones para el modelo completo, y los resultados de las 4 hipótesis propuestas.

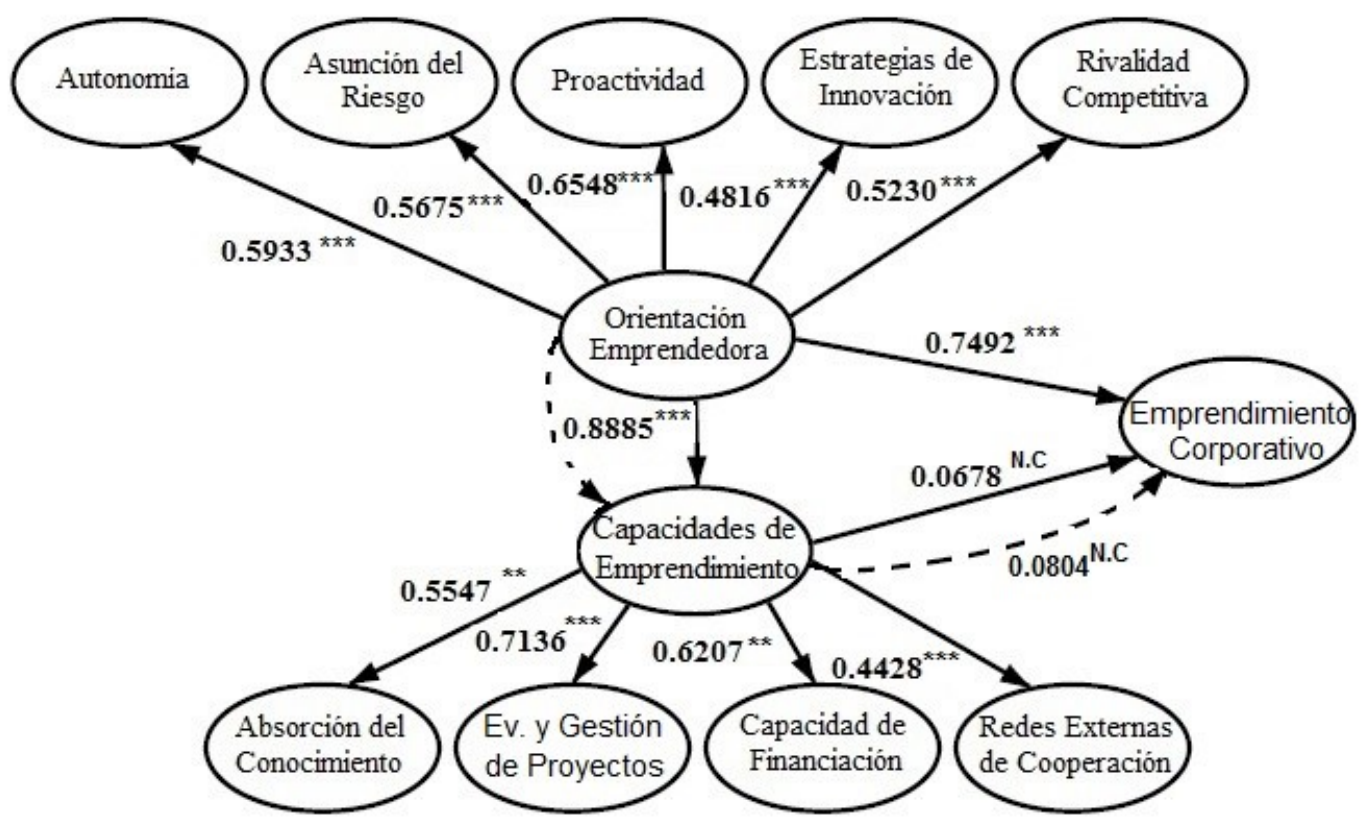

Figura 4. Estimaciones del modelo: La OE, Capacidades y su impacto en las acciones de EC Estimaciones del modelo: La OE, Capacidades y su impacto en las acciones de EC

\section{Conclusiones, recomendaciones y consideraciones finales}

La investigación se propuso clarificar la diferencia y la complementariedad entre la OE como conducta e intención y el EC como acción y comportamiento, dos constructos que con frecuencia han sido usados indiferenciadamente. Se dio respuestas a preguntas planteadas recientemente por autores seminales de la OE y el EC (Bouchard y Basso, 2011), la cual es coherente con el único estudio encontrado en esta misma línea 
REMEF (The Mexican Journal of Economics and Finance)

(Hasan, Syyedhamzeh y Ali, 2013). Este trabajo empírico, diferente a los de tipo cualitativo o descriptivo que han estudiado el fenómeno de EC en Latino América y no realizado hasta el momento para el contexto de una de las economías emergentes del continente, como lo es Colombia; con las relaciones del modelo validadas, se pudo establecer que el factor $\mathrm{OE}$ está integrado por cinco dimensiones que resultan estadísticamente significativas y coinciden con Lumpkin y Dess (1996).

Con la investigación se responden inquietudes de Miller (2011), relacionadas sobre cuáles son las capacidades que impactan favorablemente en el EC, contrastando diversas rutinas que integran un modelo multidimensional de capacidades de EC, todas ellas significativas y de impacto positivo en las acciones de emprendimiento corporativo de las PYME estudiadas: la absorción de conocimiento, redes externas de cooperación, evaluación y gestión de proyectos, capacidades de financiación; este análisis permitió establecer el constructo Capacidades de Emprendimiento Corporativo, no evidenciado en su existencia en la revisión que se realizó de la literatura, el cual integra diversas capacidades, analizadas por separado en trabajos previos.

Metodológicamente, el aporte de la propuesta radica en la realización del análisis estructural de múltiples variables determinantes de la OE y su relación con el EC, mediada por las capacidades de EC, constructo del cual se propone definición, y del que hace parte la capacidad de Evaluar y gestionar proyectos, poco explorada en estudios previos. Se logra así una imagen más integrada del fenómeno social en los contextos latinoamericano y colombiano.

Se comprueba el efecto positivo de la $\mathrm{OE}$ en el EC y también en la estructuración de capacidades de EC, lo que es un proceso lógico cuando se quiere trascender de disponer de una mentalidad emprendedora, a desarrollar y ejecutar ideas innovadoras, lo que requiere de recursos y capacidades que potencien el EC eficaz. Si bien los hallazgos del estudio y el modelo propuesto son relevantes para todo tipo de organizaciones, para las PYME puede ser más significativo, por las limitaciones en capacidades que generalmente adolecen.

Desde el punto de vista práctico, con los resultados de esta investigación, pueden ganar relevancia los fenómenos de la $\mathrm{OE}$ y EC, dado el dinamismo que genera en las organizaciones existentes, sin importar su edad, tamaño y organización jurídica. Las empresas deben tener como parte de la estrategia empresarial, el trabajar por el fortalecimiento de la OE y su penetración a toda la organización; para ello debe reconocer sus componentes, entre otros: los niveles de jerarquías de la estructura organizacional, sistemas de recompensas, autonomía y empoderamiento, formación en emprendimiento, gestión del conocimiento, cultura emprendedora, redes de trabajo colaborativo interno y externo; conocimiento profundo de las condiciones del medio externo, de las necesidades de los clientes y de la competencia.

Adicionalmente, los resultados que se logren podrán ser tenidos en cuenta por el Estado para considerar ajustes en la regulación que impulse el EC y la innovación, para apoyar con más efectividad al sector real y en particular a las PYME. Por su parte, las instituciones de educación superior, que forman profesionales en el campo de la Administración o en otras disciplinas afines, podrán disponer de los hallazgos de la investigación para complementar los diseños de los currículos para formar el espíritu emprendedor de sus educandos, formar habilidades y capacidades de emprendimiento, y mejorar los procesos de consultoría empresarial en los que intervienen. Respecto al rol fundamental de la academia en el desarrollo de la investigación-cienciatecnología y en transferencia de conocimientos al sector real, también el estudio aporta a dinamizar el triángulo Universidad-Empresa-Estado, necesario para incrementar la competitividad.

El alcance del trabajo, incluyó la capacidad de evaluación y gestión de proyectos, futuros trabajos podrían continuar con la exploración de los factores que determinan otras capacidades organizacionales, varias ya establecidas desde la teoría y otras que aún permanecen ocultas o no se han confrontado estadísticamente.

Como limitaciones de este trabajo se incluyen la diversidad de capacidades de gestión relacionadas con el EC, precisando a elegir entre algunas; la relación poco analizada con el tema de cultura empresarial enfocada en el emprendimiento innovador y con el liderazgo como competencia de las organizaciones que impulse 
modelos de gestión y sistemas de innovación gerencial; de esta manera, podrán confrontarse más capacidades de EC e integrarse en modelos multidimensionales, para analizar el papel de ser mediadoras entre la OE y el EC. También como limitante se encuentra la falta de información cuantitativa de indicadores de resultados, entre ellos los del mercado y financiera, para contrastar los impactos de la OE y del EC en el desempeño de las empresas analizadas, variables que se han estudiado en investigaciones realizadas en otros contextos; para solucionar esta falencia, se incluyeron variables resultado proveniente de las percepciones de los empresarios como medidas subjetivas, las cuales ya han sido consideradas por estudios previos sobre el impacto de la OE (Harris, 2001; Hooley, Greenleya, Cadogana y Fahy, 2005).

Para continuar el análisis conjunto de los dos fenómenos OE y EC y aportar a una mejor comprensión e interacción de los fenómenos estudiados, futuras líneas de investigación podrían dirigirse al análisis de antecedentes como el entorno, políticas públicas que favorecen el EC innovador, aspectos psicológicos, formación en emprendimiento, e impactos tanto de la OE con el desempeño, como el EC y el desempeño, en especial en AL, donde estas dos últimas relaciones no se ha contrastado en estudios cuantitativos. Sería además importante seguir corroborando el impacto de la $\mathrm{OE}$ y el EC en variables financieras y participación en el mercado.

\section{Agradecimientos}

Se agradece a la Cámara de Comercio de Medellín por suministrar la base de datos de las PYME de la región, los directivos de las empresas que aceptaron colaborar con este estudio; y en especial a la Universidad EAFIT, que financió el proyecto de investigación.

\section{Referencias}

[1] Ajzen, I. (1991). The Theory of Planned Behavior. Organizational Behavior and Human Decision Processes, 50 (2), 179-211. https://doi.org/10.1016/0749-5978(91)90020-T

[2] Álvarez, S. y Busenitz, L (2001). The entrepreneurship of resource-based theory. Journal of Management, 27, 755-775. https://doi.org/10.1177/014920630102700609

[3] Arboleda, V. (2013). Proyectos. Identificación, Formulación, Evaluación y Gerencia. Bogotá, Colombia. Alfaomega Editores.

[4] Antoncic, B. (2007). Intrapreneurship: a comparative structural equation modeling study. Industrial Management \& Data Systems. 107 (3), 309-325. https://doi.org/10.1108/02635570710734244

[5] Antoncic, B. y Hisrich, R. (2001). Intrapreneurship: Construct Refinement and Cross-Cultural Validation. Journal of Business Venturing, 16 (5), 495-527. https://doi.org/10.1016/S0883-9026(99)00054-3

[6] Baker, W, Grinstein, A. y Perin, M. (2019). The impact of entrepreneurial orientation on foreign market entry: the roles of marketing program adaptation, cultural distance, and unanticipated events. Journal of International Entrepreneurship, 1-29. https://doi.org/10.1007/s10843-019-00257-0

[7] Banco Mundial. Mente, Sociedad y Conducta. (2015). Grupo Banco Mundial. Recuperado de: http://pubdocs.worldbank.org/en/210751493925193701/pdf/Overview-Spanish.pdf

[8] Banco Mundial. Doing Business. (2019). Capacitación para las reformas. Grupo Banco Mundial. Recuperado de: https://espanol.doingbusiness.org/es/reports/global-reports/doing-business-2019

[9] Barney, J. (1991): Firm resources and sustained competitive advantage, Journal of Management, 17 (1), 99-120. https://doi.org/10.1177/014920639101700108

[10] Bartlett, M. (1974). A note on multiplying factors for various chi-squared approximations. Journal of the Royal Statistical Society, 16, 296-298. Recuperado en: https://www.scirp.org/(S(351jmbntvnsjt1aadkposzje))/reference/ReferencesPapers.aspx?ReferenceID=1565554 
[11] Barriga, E. (2006). Pyme y el acceso al crédito. Caja de herramientas para pyme. Medellín, Colombia. Edición Portafolio-Universidad EAFIT.

[12] Birkinshaw, J., Hamel, G. y Mol, M. (2008). Management innovation. Academy of Management Review, 33(4), 825-845. https://doi.org/10.5465/amr.2008.34421969

[13] Blanco, N. y Pirela, J. (2015. La complementariedad metodológica: Estrategia de integración de enfoques en la investigación social. Revista Espacios públicos. V.19, 45, 2016, 97-111. ISSN: 1665-8140. Recuperado en: https://www.redalyc.org/articulo.oa?id=67646966005

[14] Bratman, M. (1987). Intention, Plans, and Practical Reason. Cambridge, Massachusetts: Harvard University Press. https://doi.org/10.2307/2185304

[15] Brealey, R., Myers, S. y Allen, F. (2006). Principios de Finanzas Corporativas. España. Editorial Mc. Graw Hill.

[16] Bouchard, V. y Basso, O. (2011). Exploring the links between entrepreneurial orientation and intrapreneurship in SMEs. Journal of Small Business and Enterprise Development, 18 (2), 219-231. https://doi.org/10.1108/14626001111127043

[17] Cardona, R., Cardona, J. y Naranjo, A (2012). Priorización de proyectos del sector Real. Revista PODIUM, UESS, Ecuador, 21, 9-29.

[18] Cardona, R., Martins, I. y Velasquez, E. (2017). Entrepreneurial Orientation, assessment and management of projects and impact in corporate entrepreneurship: intention to action. Revista Cuadernos de Gestión, 17 (2), 36-62. DOI: $10.5295 / \mathrm{cdg} .140511 \mathrm{rc}$

[19] Cardona, R., Martins, I. y Velasquez, E. (2016). Capacidades de emprendimiento corporativo y su impacto en los proyectos innovadores. Revista Estudios Interdisciplinarios de la Organización, 10, 3-27

[20] Chetty, S. y Wilson, H. (2003). Collaborating with competitors to acquire resources. International Business Review, 12, 61-81. https://doi.org/10.1016/S0969-5931(02)00088-4

[21] Corbett, A., Covin, J., O'Connor, G. y Tucci, C. (2013). Corporate entrepreneurship: state-of-the-art research and a future research agenda. J Prod Innov Manag, 30 (5), 812-820. https://doi.org/ 10.1111/jpim.12031

[22] Covin, J. y Wales, W. (2019). Crafting high-impact entrepreneurial orientation research: Some suggested guidelines. Entrepreneurship Theory and Practice, 43 (1), 3-18. https://doi.org/10.1177/1042258718773181

[23] Covin, J. y Lumpkin, G. (2011). Entrepreneurial Orientation Theory and Research: Reflections on a Needed Construct. Entrepreneurship Theory and Practice, 855-872. https://doi.org/10.1111/j.1540-6520.2011.00482.x

[24] Covin, J. y Wales, W. (2011). The Measurement of Entrepreneurial Orientation. Entrepreneurship: Theory and Practice, 1-26. https://doi.org/10.1111/j.1540-6520.2010.00432.x

[25] Covin, J. y Slevin, D. (1991). A Conceptual model of entrepreneurship as firm behavior. Entrepreneurship: Theory and Practice, 16, 7-25. https://doi.org/10.1177/104225879101600102

[26] Dess, G. y Lumpkin, G (2005). The Role of Entrepreneurial Orientation in Stimulating Effective Corporate Entrepreneurship. The Academy of Management Executive, 146-157. https://doi.org/10.5465/ame.2005.15841975

[27] Foro económico mundial-FEM. (2018). The Global Competitiveness Report.

[28] Gelderen, M, Kautonen, T y Fink, M. (2015). From Entrepreneurial Intentions to Actions: Self-Control and Action-Related Doubt, Fear, and Aversion. Journal of Business Venturing. 30, 655-673. DOI: 10.1016/j.jbusvent.2015.01.003

[29] Global Entrepreneurship Monitor-GEM, 2018/2019 Global Report, 20o. Aniversario (2018). Recuperado en: https://www.gemconsortium.org/report/gem-2018-2019-global-report

[30] Global Entrepreneurship Monitor. Global Entrepreneurship Monitor-GEM España. (2014). Cantabria, España. Editorial de la Universidad de Cantabria.

[31] Goodale, J., Kuratko, D., Hornsby, J. y Covin, J. (2011). Operations management and corporate entrepreneurship: The moderating effect of operations control on the antecedents of corporate entrepreneurial activity in relation to innovation performance. Journal of Operations Management, 29 (2), 116-127. https://doi.org/10.1016/j.jom.2010.07.005 
[32] Grant, R. (2006). Dirección estratégica: conceptos, técnicas y aplicaciones. Madrid. Epaña. Editorial Civitas.

[33] Grant, R. (1996). Toward a Knowledge-based theory of the firm. Strategic Management Journal, 17, $109-122$. https://doi.org/10.1002/smj.4250171110

[34] Gulati, R. (1998). Alliances and networks. Strategic Management Journal, $19,293-317$. https://doi.org/10.1002/(SICI)1097-0266(199804)19:4<293::AID-SMJ982>3.0.CO;2-M

[35] Hasan, K., Syyedhamzeh, N. y Ali, F. (2013). The influence of entrepreneurial orientation on innovative performance. Journal of Knowledge-based Innovation in China, 5 (3), 262 - 278. https://doi.org/10.1108/JKIC-092013-0017

[36] Harris, L. (2001). Market orientation and performance: objective and subjective empirical evidence from UK companies. Journal of Management Studies, 38, 17-43. https://doi.org/10.1111/1467-6486.00226

[37] Hoang, H. y Antoncic, B. (2003). Network-based research in entrepreneurship: a critical review. Journal of Business Venturing, 18, 165-187. https://doi.org/10.1016/S0883-9026(02)00081-2

[38] Hooley, G., Greenleya, G., Cadogana, J. y Fahy; J. (2005). The performance impact of marketing resources. Journal of Business Research, 58, 18-27. https://doi.org/10.1016/S0148-2963(03)00109-7

[39] Indice Global de Innovation (2018). Energizing the Word with innovation. Chapter 1: The Global Innovation Index 2018: Energizing the World with Innovation. NY, U.S.A. Ed. Johnson Cornell University. INSEAD; WIPO.

[40] Indice Global de Innovation (2014). The Human Factor in Innovation. Chapter 2: The Human Factor in Innovation, 69-72. NY, U.S.A. Ed. Johnson Cornell University. INSEAD; WIPO.

[41] Ireland, R, Covin, J. y Kuratko, D. (2009). Conceptualizing corporate entrepreneurship strategy. Entrepreneurship Theory and Practice, 33(1), 19-47. https://doi.org/10.1111/j.1540-6520.2008.00279.x

[42] Ireland, R., Kuratko, D., y Morris, M. (2006). A health audit for corporate entrepreneurship: innovation at all levels: part I. Journal of Business Strategy, 27 (1), 10-17. https://doi.org/10.1108/02756660610640137

[43] Kaiser, H. (1958). The varimax criterion for analytic rotation in factor analysis. Psychometrika, 23(3), $187-200$. https://doi.org/10.1007/BF02289233

[44] Kirzner, I. 1973. Competition and Entrepreneurship. Chicago: University of Chicago Press.

[45] Kuratko, D. y Audretsch, D. (2009). Strategic Entrepreneurship: Exploring Perspectives an emerging Concept. Entrepreneurship: Theory and Practice, 33 (1), 1-17. https://doi.org/10.1111/j.1540-6520.2008.00278.x

[46] Lumpkin, G. y Dess, G. (1996). Clarifying the entrepreneurial orientation construct and linking it to performance. Academy of Management Review, 21 (1), 135-173. https://doi.org/10.5465/amr.1996.9602161568

[47] Mcfadzean, E., O'Loughlin, A. y Shaw, E. (2005). Corporate Entrepreneurship and Innovation Part 1: The missing link. European Journal of Innovation Management, 8 (3), 350-372. https://doi.org/10.1108/14601060510610207

[48] Martins, I., Rialp, A., Rialp, J. y Rocio A. (2015). El Uso de las Redes como Propulsor para la Orientación Emprendedora y el Crecimiento de la PYME. Innovar, 55, 113-126. http://dx.doi.org/10.15446/innovar.v25n55.47227.

[49] Meade, A., Watson, A. y Kroustalis, M. (2007). Assessing common methods bias in organizational research. 22nd Annual Meeting of the Society for Industrial and Organizational Psychology Proceedings, New York, April. http://citeseerx.ist.psu.edu/viewdoc/download?doi=10.1.1.457.5262\&rep $=$ rep1\&type $=$ pdf

[50] Miller, D. (2011). Miller (1983) Revisited: A Reflection on EO Research and Some Suggestions for the Future. Entrepreneurship Theory and Practice, 873-894. https://doi.org/10.1111/j.1540-6520.2011.00457.x

[51] Miller, D. (1983). The correlates of entrepreneurship in three types of firms. Management Science, (29), $770-791$. https://doi.org/10.1287/mnsc.29.7.770

[52] Mintzberg, H. (1973). Strategic, making in three models. California Management Review, (16), 44-53. https://doi.org/10.2307/41164491

[53] Mintzberg, H. (1994). The Rise and Fall of Strategic Planning. México, México. Editorial Omega. 
REMEF (The Mexican Journal of Economics and Finance)

[54] Nonaka, L. y Takeuchi, H. (1995). The Knowledge-Creating Company: How Japanese Companies Create the Dynamics Innovation. New York, U.S.A. Oxford University Press.

[55] OCDE- Foro Consultivo Científico y Tecnológico, 2012. La Estrategia de Innovación de la OCDE: empezar hoy el mañana, 95-130, Paris: Organización para la Cooperación y el Desarrollo Económicos. http: //www.foroconsultivo.org.mx/libros_editados/estrategia_innovacion_ocde.pdf

[56] OCDE. (2005). Manual de Oslo. Guía para la recogida e interpretación de datos de innovación, Madrid (Ed.) Organización para la Cooperación y el Desarrollo Económicos y Eurostat. Paris: Grupo Tragsa. http://www.itq.edu.mx/convocatorias/manualdeoslo.pdf

[57] Pérez, C. (2008). Econometría Avanzada: técnicas y aplicaciones. 1ª Edición. Prentice Hall. Madrid.

[58] Pinchot III Gifford. (1985). Intrapreneuring. Whay you don't have to leave the corporation to become an Entrepreneur. 1a. Edición. New York, U.S.A. Harper \& Row, Publishers.

[59] Podsakoff, P., MacKenzie, S., Lee, J., Podsakoff, N. (2003). Common method biases in behavioral research: a critical review of the literature and recommended remedies. Journal of Applied Psychology, (88), 879-903. DOI: 10.1037/0021-9010.88.5.879

[60] Rauch, A., Wiklund, J., Lumpkin, G., \& Frese, M. (2009). Entrepreneurial orientation and business performance: an assessment of past research and suggestions for the future. Entrepreneurship: Theory and Practice, 33 (3), 761-787. https://doi.org/10.1111/j.1540-6520.2009.00308.x

[61] Rezaei, J. y Ortt, R. (2018). Entrepreneurial orientation and firm performance: the mediating role of functional performances. Management Research Review, Emerald, 41 (7), 878-900. https://doi.org/10.1108/MRR-03-20170092

[62] Rosenbusch, N., Rauch, A. y Bausch, A. (2013). The mediating role of entrepreneurial orientation in the task environment-performance relationship: a meta-analysis. Journal of Management, 39 (3), $633-659$. https://doi.org/10.1177/0149206311425612

[63] Rosillo, J. (2008). Formulación y Evaluación de Proyectos de Inversión para Empresas Manufactureras y de Servicios. Primera Edición, Bogotá, Colombia. Cengage Learning.

[64] Sapag, N., Sapag, R., Sapag, J. (2014). Preparación y Evaluación de Proyectos. Sexta edición, México, México. Ed. McGraw Hill.

[65] Sapienza, H., De Clercq, D. y Sandberg, W. (2005). Antecedents of international and domestic learning effort. Journal of Business Venturing, 20, 437-457. https://doi.org/10.1016/j.jbusvent.2004.03.001

[66] Schlaegel, C. y Koenig, M. (2014). Determinants of entrepreneurial intent: a meta-analytic test and integration of competing models. Entrepreneurship Theory and Practice. 38, 291-332. DOI: 10.1111/etap.12087

[67] Schumpeter, J. (1942). The Process of Creative Destruction. (Capítulo, VII) y Crumbling Walls (1975). Capitalism, Socialism and Democracy. (Cap. XII). NY, U.S.A 1975. Ed. Harper \& Row Publishers

[68] Sharma, P. y Chrisman, J. (1999). Toward a reconciliation of definitional issues in the field of corporate entrepreneurship. Entrepreneurship Theory and Practice, 31, 11-27. https://doi.org/10.1007/978-3-540-48543-8_4

[69] Stevenson, H. y Jarillo, J. (1990). A paradigm of entrepreneurship: Entrepreneurial Management. Strategic Management Journal, V. 11, 17-27. https://doi.org/10.1007/978-3-540-48543-8_7

[70] Stambaugh, J.E., Martinez, J., Lumpkin, G.T. y Kataria, N. (2017). How well do EO measures and entrepreneurial behavior match? International Entrepreneurship and Management Journal, V. 13, 3, 717-737. https://doi.org/10.1007/s11365-016-0432-5

[71] Taheri, B., Bititci, U., Gannon, M. J. y Cordina, R. (2019). Investigating the influence of performance measurement on learning, entrepreneurial orientation and performance in turbulent markets. International Journal of Contemporary Hospitality Management, V. 31, 3, 1224-1246. https://doi.org/10.1108/IJCHM-11-2017-0744

[72] Teece, D. J., Pisano, G. y Schuen, A. (1997): Dynamic capabilities and strategic management. Strategic Management Journal, 18 (7), 509-533. https://doi.org/10.1002/(SICI)1097-0266(199708)18:7<509::AIDSMJ882>3.0.CO;2-Z 
[73] Titus Jr, V., Parker, O. y Covin, J. (2019). Organizational Aspirations and External Venturing: The Contingency of Entrepreneurial Orientation. Entrepreneurship Theory and Practice, 00(0) 1-26, DOI: 1042258719838473.

[74] Todorovic, Z., Todorovic, D. y Ma, J. (2015). Corporate entrepreneurship and entrepreneurial orientation in corporate environment: A discussion. Academy of Entrepreneurship Journal, 21, 1, 82-92.

[75] Urbano, D., Toledano, N. y Soriano, D. (2010). Prácticas de gestión de recursos humanos y desarrollo de nuevos proyectos innovadores: Un estudio de casos en las PYME. Universia Business Review, 29,116-130. https://dialnet.unirioja.es/servlet/articulo?codigo $=3435835$

[76] Vélez C., J. I. (2012). Intention in intervention: a philosophical, theoretical and empirical exploration. Tesis (Dr.), Victoria University of Wellington. http://hdl.handle.net/10063/2210.

[77] Wang, C.L. (2008). Entrepreneurial orientation, learning orientation, and firm performance. Entrepreneurship Theory and Practice, 32 (4), 635-657. https://doi.org/10.1111/j.1540-6520.2008.00246.x

[78] Wales, W.J. (2016). Entrepreneurial orientation: a review and synthesis of promising research directions. International Small Business Journal, 34 (1), 3-15. https://doi.org/10.1177/0266242615613840

[79] Wernerfelt, B. (1984). A resource based view of the firm. Strategic Management Journal, 5, 171-180. https://doi.org/10.1111/j.1540-6520.2011.00451.x

[80] Wiklund, J.; Shepherd, D. (2003). Knowledge-Based Resources, Entrepreneurial Orientation, and the Performance of Small and Medium-Sized Businesses. Strategic Management Journal, (24), 1307-314. https://doi.org/10.1002/smj.360

[81] Zahra, S. y George, G. (2002). Absorptive capacity: A review, reconcepualisation, and extension. Academy of Management Review, 27 (2), 185-203. https://doi.org/10.5465/amr.2002.6587995

[82] Zahra, S. (1993). Environment, corporate entrepreneurship and financial performance: a taxonomic approach. Journal of Business Venturing, 8, 319-340. https://doi.org/10.1016/0883-9026(93)90003-N

[83] Zhang, Y., Khan, U., Lee, S., y Salik, M. (2019). The Influence of Management Innovation and Technological Innovation on Organization Performance. A Mediating Role of Sustainability. SUSTAINABILITY, 11(2). https://doi.org/10.3390/su11020495 
ANEXOS Anexo 1. Preguntas de la encuesta (1/2)

\begin{tabular}{|c|c|c|c|}
\hline 26 & & & ORIENTACIÓN EMPRENDEDORA \\
\hline & No. & & PROACTIVIDAD \\
\hline & 1 & oe1 & $\begin{array}{l}\text { La empresa no mantiene su modelo tradicional de negocio y ve la necesidad de implementar } \\
\text { acciones innovadoras frente al mercado }\end{array}$ \\
\hline & 2 & oe2 & $\begin{array}{l}\text { En la empresa se implementan cambios que afectan el desempeño del sector, generalmente } \\
\text { antes que la competencia, en lugar de ser reactiva para contrarrestar las decisiones de los } \\
\text { competidores }\end{array}$ \\
\hline & 3 & oe3 & $\begin{array}{l}\text { Normalmente, los nuevos productos puestos en el mercado, han sido desarrollados por la } \\
\text { empresa más que introducidas por otras compañías }\end{array}$ \\
\hline & & & ASUNCION DEL RIESGO \\
\hline & 4 & oe4 4 & $\begin{array}{l}\text { Preferiblemente, la empresa desarrolla iniciativas en negocios arriesgados y desconocidos } \\
\text { pero que podrian tener alta rentabilidad }\end{array}$ \\
\hline & 5 & oes & $\begin{array}{l}\text { Cuando se enfrenta a la toma de decisiones en situaciones de incertidumbre, su empresa } \\
\text { opta por aprovechar prontamente las oportunidades, en lugar de esperar y actuar con cautela } \\
\text { para evitar costosas equivocaciones }\end{array}$ \\
\hline & 6 & oe6 & $\begin{array}{l}\text { Debido al entorno cambiante, es mejor explorarlo audaz y arriesgadamente en lugar de hacerlo } \\
\text { de forma gradual y tímidamente }\end{array}$ \\
\hline & & & ESTRATEGIAS DE INNOVACIÓN \\
\hline & 7 & oe7 & La empresa ha modificado su estructura organizacional para incrementar la innovación \\
\hline & 8 & oe8 & $\begin{array}{l}\text { Los recursos que destina la compañía a l+D crecen anualmente en forma significativa, en } \\
\text { relación al crecimiento de los ingresos de la empresa y a los presupuestos del sector en este } \\
\text { rubro }\end{array}$ \\
\hline & 9 & oeg & $\begin{array}{l}\text { La innovación está dispersa en toda la organización y no centralizada en un área determinada } \\
\text { de la empresa }\end{array}$ \\
\hline & 10 & oe10 & $\begin{array}{l}\text { Los nuevos productos desarrollados por la empresa corresponden a cambios significativos y } \\
\text { no a mejoras de los ya existentes en el mercado }\end{array}$ \\
\hline & 11 & oe11 & $\begin{array}{l}\text { La implementación de nuevas tecnologías corresponden más a transformaciones radicales } \\
\text { que a mejoras en las ya existentes }\end{array}$ \\
\hline & 12 & oe12 & Las nuevas tecnologías han sido desarrolladas por la empresa y no adquiridas en el mercado \\
\hline & 13 & oe13 & $\begin{array}{l}\text { La empresa innova en forma permanente en los procesos administrativos y en sistemas de } \\
\text { información }\end{array}$ \\
\hline & 14 & oe14 & $\begin{array}{l}\text { La estrategia de la compañía se enfoca más en competir por la diferenciación (relacionada } \\
\text { con los atributos del producto, calidad, innovación, Investigación y desarrollo y búsqueda de } \\
\text { nuevos nichos de mercados) que en liderazgo en costos y precios bajos (mejorar eficiencias) }\end{array}$ \\
\hline & & & CONDICIONES DEL ENTORNO Y RIVALIDAD COMERCIAL \\
\hline & 15 & oe15 & $\begin{array}{l}\text { Dado al ambiente externo inseguro e inestable en el que se mueve la empresa, } \\
\text { constantemente se cambian las estrategias de mercadeo }\end{array}$ \\
\hline & 16 & oe16 & Las acciones de los competidores son bastante difíciles de predecir \\
\hline & 17 & oe17 & La rivalidad del sector es muy alta y se da una fuerte agresividad competitiva \\
\hline & 18 & oe18 & El ritmo al que los productos y/o servicios se vuelven obsoletos en el sector es alto \\
\hline & 19 & oe19 & La demanda y gusto de los consumidores son bastante difíciles de prever \\
\hline & 20 & oe20 & El desarrollo tecnológico en la industria o sector es alto \\
\hline & & & AUTONOMIA \\
\hline & 21 & oe21 & $\begin{array}{l}\text { La empresa tiene incentivos para los empleados emprendedores que generen ideas y } \\
\text { nuevos proyectos, como beneficios, compensaciones financieras, premios, reconocimiento y } \\
\text { oportunidades de ascenso }\end{array}$ \\
\hline & 22 & $0 \mathrm{e} 22$ & En general, el nivel de espíritu emprendedor en los empleados de la organización es alto \\
\hline & 23 & oe23 & $\begin{array}{l}\text { Se incentiva a los individuos y equipos a romper paradigmas en lugar de seguir los procesos y } \\
\text { estrategias estandarizadas como base de decisión }\end{array}$ \\
\hline & 24 & oe24 & $\begin{array}{l}\text { Las relaciones internas de la compañía, el trabajo en equipo, la comunicación y la } \\
\text { cooperación, favorecen notablemente el emprendimiento colaborativo }\end{array}$ \\
\hline & 25 & oe25 & $\begin{array}{l}\text { En la firma se tolera el fracaso, ante los cambios propuestos y resultados de las ideas } \\
\text { implementadas por los empleados }\end{array}$ \\
\hline & 26 & oe26 & $\begin{array}{l}\text { Las relaciones jerárquicas de la empresa son flexibles y se ajustan a las necesidades de } \\
\text { emprendimiento y de innovación. }\end{array}$ \\
\hline
\end{tabular}


Preguntas de la encuesta $(2 / 2)$

\begin{tabular}{|c|c|c|c|}
\hline 22 & & & CAPACIDADES DE EMPRENDIMIENTO CORPORATIVO \\
\hline 6 & & & CONOCIMIENTO PARA EL EMPRENDIMIENTO \\
\hline & 27 & rc1 & Se promueve el conocimiento, realizando constantemente capacitación de los empleados \\
\hline & 28 & rc2 & $\begin{array}{l}\text { El conocimiento de la empresa es bastante complejo, difícil de identificar y comprender, aún } \\
\text { con experiencia personal }\end{array}$ \\
\hline & 29 & $\mathrm{rc} 3$ & Existen mecanismos formales para generar, integrar y transferir el conocimiento \\
\hline & 30 & rc4 & El conocimiento está documentado a través de manuales, reportes y documentos escritos \\
\hline & 31 & rc5 & Los nuevos conocimientos técnicos se aplican rápidamente en la organización \\
\hline & 32 & rc6 & $\begin{array}{l}\text { El desarrollo del conocimiento ha permitido a la empresa identificar y seleccionar } \\
\text { oportunidades de negocios, provenientes del mercado y de los cambios tecnológicos }\end{array}$ \\
\hline 8 & & & CAPACIDAD DE EVALUAR Y GESTIONAR PROYECTOS \\
\hline & 33 & rc7 & $\begin{array}{l}\text { Los mandos medios y de nivel superior han recibido capacitación en formulación, evaluación, } \\
\text { y dirección de proyectos }\end{array}$ \\
\hline & 34 & rc8 & $\begin{array}{l}\text { Existe un funcionario o área en la compañía, que desempeñe las funciones relativas a evaluar } \\
\text { y a consolidar en el plan financiero a mediano plazo los proyectos aprobados, para determinar } \\
\text { el presupuesto de inversión y las necesidades de financiación }\end{array}$ \\
\hline & 35 & rc9 & $\begin{array}{l}\text { La compañía evalúa financieramente las decisiones de inversión, bajo las metodologías de } \\
\text { flujos de caja, determinando criterios financieros como la TIR y el VPN }\end{array}$ \\
\hline & 36 & rc10 & $\begin{array}{l}\text { Se tiene claramente establecida la tasa anual que deben rentar las inversiones que la } \\
\text { empresa realiza }\end{array}$ \\
\hline & 37 & rc11 & $\begin{array}{l}\text { En la compañía se conoce a cuánto asciende el costo de capital (conocido por su sigla en } \\
\text { inglés como el WACC o Weight average capital cost) }\end{array}$ \\
\hline & 38 & rc12 & Se tienen procesos de seguimiento y control formal a los proyectos que se implementan \\
\hline & 39 & rc13 & $\begin{array}{l}\text { Se siguen metodologías formales para priorizar proyectos -dados los múltiples proyectos } \\
\text { resultantes de los procesos del emprendimiento corporativo y no basadas solamente en la } \\
\text { limitante del presupuesto de inversiones }\end{array}$ \\
\hline & 40 & rc14 & Se goza de una extensa experiencia laboral en el sector en el que se ubica la empresa \\
\hline 5 & & & CAPACIDADES DE FINANCIACIÓN \\
\hline & 41 & rc15 & $\begin{array}{l}\text { La disponibilidad de capital no es insuficiente y no es impedimento para el desarrollo } \\
\text { empresarial satisfactorio }\end{array}$ \\
\hline & 42 & rc16 & $\begin{array}{l}\text { Ante proyectos atractivos y considerados factibles financieramente, la empresa tiene facilidad } \\
\text { en acceder a financiación adicional (dueños, amigos, familiares, entidades financieras) }\end{array}$ \\
\hline & 43 & rc17 & $\begin{array}{l}\text { Los dueños tienen disposición y capacidad de incrementar el capital, cuando consideran que } \\
\text { existen oportunidades de negocios y proyectos de inversión atractivos }\end{array}$ \\
\hline & 44 & rc18 & $\begin{array}{l}\text { Ante proyectos atractivos y considerados factibles financieramente, la empresa tiene facilidad } \\
\text { en acceder a financiación adicional con terceros (amigos, familiares, entidades financieras) }\end{array}$ \\
\hline & 45 & rc19 & $\begin{array}{l}\text { La empresa ha podido acceder a recursos de financiación para implementar proyectos de } \\
\text { inversión, provenientes de BANCÓLDEX, avales de FOGAFIN o fuentes de financiación } \\
\text { COLCIENCIAS e INNPULSA }\end{array}$ \\
\hline 3 & 46 & & TRABAJO EN REDES EXTERNAS DE COOPERACIÓN \\
\hline & 47 & rc20 & $\begin{array}{l}\text { La organización ha desarrollado relaciones externas, comunicación permanente y alianzas } \\
\text { estratégicas con clientes, proveedores y competidores, que posibilitan el emprendimiento } \\
\text { conjunto }\end{array}$ \\
\hline & 48 & rc21 & $\begin{array}{l}\text { El trabajo en estas redes externas de colaboración ha permitido el análisis de los efectos del } \\
\text { entorno e identificación de oportunidades de innovación, mejoras en competitividad y } \\
\text { agregación de valor }\end{array}$ \\
\hline & 49 & rc22 & $\begin{array}{l}\text { La firma participa en redes Universidad -Empresa - Estado y éstas han favorecido la } \\
\text { investigación y el desarrollo, así como el emprendimiento corporativo }\end{array}$ \\
\hline 7 & & & $\begin{array}{l}\text { ACCIONES DE EMPRENDIMIENTO CORPORATIVO: NUEVOS PRODUCTOS, TECNOLOGIAS Y } \\
\text { NUEVOS NEGOCIOS }\end{array}$ \\
\hline & 50 & ec1 & Muchos proyectos pasan de la etapa de desarrollo y llegan a ser éxitos comerciales \\
\hline & 51 & ec2 & La empresa ha invertido recursos significativos en los últimos tres años en el desarrollo de productos \\
\hline & 52 & ec3 & $\begin{array}{l}\text { Los ingresos por ventas correspondientes a nuevos productos han ganado participación en los ingresos } \\
\text { totales, durante los últimos tres años }\end{array}$ \\
\hline & 53 & ec4 & En los últimos tres años, nuevas tecnologías se han implementado \\
\hline & 54 & rc5 & $\begin{array}{l}\text { La empresa ha invertido recursos significativos en los últimos tres años, en la creación de nuevos } \\
\text { negocios, diferentes a los que han sido su tradición }\end{array}$ \\
\hline & 55 & rc6 & $\begin{array}{l}\text { Las ventas o ingresos correspondientes a nuevos negocios, han ganado participación en los ingresos } \\
\text { totales, durante los últimos tres años }\end{array}$ \\
\hline & 56 & rc7 & $\begin{array}{l}\text { En los últimos tres años, los ingresos de negocios internacionales han ganado participación en los } \\
\text { ingresos totales (correspondientes a exportación, importación e inversión en el exterior) }\end{array}$ \\
\hline
\end{tabular}


REMEF (The Mexican Journal of Economics and Finance)

Anexo 2. Tabla de estadística descriptiva por variables de las distintas dimensiones CARACTERÍSTICAS DE LA FIRMA TENIENDO EN CUENTA LAS CINCO DIMENSIONES DE ORIENTACIÓN EMPRENDEDORA

\begin{tabular}{|lcr|}
\hline Característica & Número de empresas con calificación superior & Porcentaje \\
\hline Orientación emprendedora & 62 & \\
\hline & & 45.3 \\
\hline Proactividad & 61 & 44.5 \\
\hline Asunción del riesgo & 72 & 52.6 \\
\hline Estrategias de Innovación & 91 & 66.4 \\
\hline Condiciones del entorno y rivalidad comercial & 67 & 48.9 \\
\hline Autonomía & 69 & 50.4 \\
\hline
\end{tabular}

\begin{tabular}{|lcr|}
\hline \multicolumn{4}{|l|}{ CARACTERÍSTICAS DE LA FIRMA SEGÚN RECURSOS Y CAPACIDADES } & & \\
\hline & & \\
\hline Característica & Número de empresas con calificación superior & Porcentaje \\
\hline Recursos y Capacidades & 69 & 50.4 \\
\hline & 70 & 51.0 \\
\hline Absorción del conocimiento & 73 & 53.3 \\
\hline Capacidades de Formulación y Gestión de Proyectos & 85 & 62.0 \\
\hline Capacidad de Financiación & 89 & 64.96 \\
\hline Redes Externas de Cooperación & &
\end{tabular}

